\title{
INSTABILITY OF ONE GLOBAL TRANSONIC SHOCK WAVE FOR THE STEADY SUPERSONIC EULER FLOW PAST A SHARP CONE
}

\author{
GANG XU AND HUICHENG YIN
}

\begin{abstract}
In this paper, we are concerned with the instability problem of one global transonic conic shock wave for the supersonic Euler flow past an infinitely long conic body whose vertex angle is less than some critical value. This is motivated by the following descriptions in the book Supersonic Flow and Shock Waves by Courant and Friedrichs: if there is a supersonic steady flow which comes from minus infinity, and the flow hits a sharp cone along its axis direction, then it follows from the Rankine-Hugoniot conditions, the physical entropy condition, and the apple curve method that there will appear a weak shock or a strong shock attached at the vertex of the cone, which corresponds to the supersonic shock or the transonic shock, respectively. A long-standing open problem is that only the weak shock could occur, and the strong shock is unstable. However, a convincing proof of this instability has apparently never been given. The aim of this paper is to understand this. In particular, under some suitable assumptions, because of the essential influence of the rotation of Euler flow, we show that a global transonic conic shock solution is unstable as long as the related sharp circular cone is perturbed.
\end{abstract}

\section{$\S 1$. Introduction and main results}

If there is a uniform supersonic flow $\left(\rho_{0}, 0,0, q_{0}, P_{0}\right)$ which comes from minus infinity, and the flow hits the circular cone $\sqrt{x_{1}^{2}+x_{2}^{2}}=b_{0} x_{3}$ along the axis $x_{3}$-direction, when the vertex angle $2 \arctan b_{0}$ of the cone is less than a critical value $\theta^{*}$, the following conic shock problems are illustrated in [6, pages 313-314, 414]: it follows from the Rankine-Hugoniot conditions and the physical entropy condition that there will appear a weak or a strong self-similar shock attached at the vertex of the cone in terms of the different

Received July 24, 2009. Revised November 22, 2009. Accepted February 1, 2010.

2000 Mathematics Subject Classification. 35L70, 35L65, 35L67, 76N15.

This project was supported by National Natural Science Foundation of China grants no. 10931007 and no. 10571082 and by National Basic Research Program of China grant no. 2006CB805902.

(C) 2010 by The Editorial Board of the Nagoya Mathematical Journal 
pressure states at infinity behind the shock surface, which corresponds to the supersonic shock or the transonic shock, respectively. A long-standing open problem is that only the weak shock could occur, and the strong shock is unstable. However, a convincing proof of this instability has apparently never been given. The aim of this paper is to understand this. More concretely, under some suitable assumptions, because of the essential influence of the rotation of Euler flow, we show that a global transonic conic shock is actually unstable when the surface of the conic body is perturbed.

The steady full compressible Euler system is described as

$$
\left\{\begin{array}{l}
\sum_{j=1}^{3} \partial_{j}\left(\rho u_{j}\right)=0, \\
\sum_{j=1}^{3} \partial_{j}\left(\rho u_{i} u_{j}\right)+\partial_{i} P=0, \\
\sum_{j=1}^{3} \partial_{j}\left(\left(\rho e+\frac{1}{2} \rho|u|^{2}+P\right) u_{j}\right)=0,
\end{array} \quad i=1,2,3\right.
$$

where $\rho, u=\left(u_{1}, u_{2}, u_{3}\right)$; and $P, e$, and $S$ stand for the density, velocity, pressure, internal energy, and specific entropy, respectively. Moreover, the pressure function $P=P(\rho, S)$ and the internal energy function $e=e(\rho, S)$ are smooth in their arguments, which satisfy $\partial_{\rho} P(\rho, S)>0$ and $\partial_{S} e(\rho, S)>0$ for $\rho>0$. In addition, $c(\rho, S)=\sqrt{\partial_{\rho} P(\rho, S)}$ stands for the sound speed.

For the ideal polytropic gases, the equations of state are given by

$$
P=A \rho^{\gamma} e^{\frac{S}{c_{v}}} \quad \text { and } \quad e=\frac{P}{(\gamma-1) \rho},
$$

where $A, c_{v}$, and $\gamma$ are positive constants and $1<\gamma<3$ (especially $\gamma \approx 1.4$ with respect to the air).

It is assumed that there is a uniform supersonic incoming flow with the constant state $\left(\rho_{0}, 0,0, q_{0}, P_{0}\right)$, and that the flow hits the perturbed conic body along the $x_{3}$-direction, whose surface equation is denoted by $r=b\left(x_{3}\right)$, where $r=\sqrt{x_{1}^{2}+x_{2}^{2}}, b\left(x_{3}\right)=b_{0} x_{3}+\varepsilon \varphi\left(x_{3}\right)$, and $\varepsilon>0$ is a small constant, $\varphi\left(x_{3}\right) \in C_{0}^{\infty}(0, l)$ with some fixed positive number $l>0$. In particular, we point out that $b_{0}>0$ is less than some critical value $b^{*}$ so that the resulted shock will attach at the vertex of the conic body (see Figure 1).

Because of the symmetric property of the perturbed conic surface, it is convenient to introduce the following cylindrical coordinates $\left(x_{3}, r\right)$ to study our problem:

$$
x_{3}=x_{3}, \quad r=\sqrt{x_{1}^{2}+x_{2}^{2}}
$$




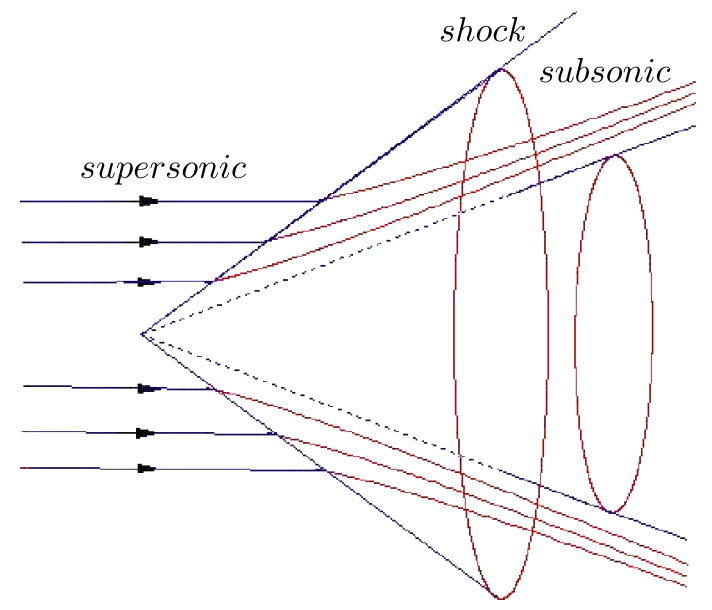

Figure 1: The transonic conic shock for the supersonic flow past a sharp cone

For the polytropic gas and axisymmetric solution to (1.1), which has the form $\quad\left(\rho(x), u_{1}(x), u_{2}(x), u_{3}(x), P(x)\right) \equiv\left(\rho\left(x_{3}, r\right), U\left(x_{3}, r\right)\left(x_{1} / r\right), U\left(x_{3}, r\right)\right.$ $\left.\left(x_{2} / r\right), u_{3}\left(x_{3}, r\right), P\left(x_{3}, r\right)\right),(1.1)$ can be reduced into

$$
\left\{\begin{array}{l}
\partial_{r}(r \rho U)+\partial_{3}\left(r \rho u_{3}\right)=0 \\
\partial_{r}\left(r \rho U^{2}\right)+\partial_{3}\left(r \rho U u_{3}\right)+r \partial_{r} P=0 \\
\partial_{r}\left(r \rho U u_{3}\right)+\partial_{3}\left(r \rho u_{3}^{2}\right)+r \partial_{r} P=0
\end{array}\right.
$$

and

$$
\frac{1}{2}\left(U^{2}+u_{3}^{2}\right)+\frac{\gamma P}{(\gamma-1) \rho}=\frac{1}{2} q_{0}^{2}+\frac{\gamma P_{0}}{(\gamma-1) \rho_{0}} \equiv C_{0} .
$$

Suppose that the flow field behind the possible shock $r=\chi\left(x_{3}\right)$ is denoted by $\left(\rho^{+}\left(x_{3}, r\right), U^{+}\left(x_{3}, r\right), u_{3}^{+}\left(x_{3}, r\right), P^{+}\left(x_{3}, r\right)\right)$. Then, in the domain $\Omega_{+} \equiv$ $\left\{\left(x_{3}, r\right): x_{3}>0, b\left(x_{3}\right)<r<\chi\left(x_{3}\right)\right\},\left(\rho^{+}, U^{+}, u_{3}^{+}, P^{+}\right)$satisfies:

$$
\left\{\begin{array}{l}
\partial_{r}\left(r \rho^{+} U^{+}\right)+\partial_{3}\left(r \rho^{+} u_{3}^{+}\right)=0 \\
\partial_{r}\left(r \rho^{+}\left(U^{+}\right)^{2}\right)+\partial_{3}\left(r \rho^{+} U^{+} u_{3}^{+}\right)+r \partial_{r} P^{+}=0 \\
\partial_{r}\left(r \rho^{+} U^{+} u_{3}^{+}\right)+\partial_{3}\left(r \rho^{+}\left(u_{3}^{+}\right)^{2}\right)+r \partial_{3} P^{+}=0 \\
\frac{1}{2}\left(\left(U^{+}\right)^{2}+\left(u_{3}^{+}\right)^{2}\right)+\frac{\gamma P^{+}}{(\gamma-1) \rho^{+}}=C_{0} .
\end{array}\right.
$$


On the shock $r=\chi\left(x_{3}\right)$, the Rankine-Hugoniot conditions imply

$$
\left\{\begin{array}{l}
{[\rho U]-\chi^{\prime}\left(x_{3}\right)\left[\rho u_{3}\right]=0,} \\
{\left[P+\rho U^{2}\right]-\chi^{\prime}\left(x_{3}\right)\left[\rho U u_{3}\right]=0} \\
{\left[\rho U u_{3}\right]-\chi^{\prime}\left(x_{3}\right)\left[P+\rho u_{3}^{2}\right]=0 .}
\end{array}\right.
$$

Meanwhile, the physical entropy condition (see [6]) holds true:

$$
P_{0}<P^{+}\left(x_{3}, \chi\left(x_{3}\right)\right)
$$

Because of the fixed wall condition, we have on the conic surface $r=b\left(x_{3}\right)$

$$
U^{+}=b^{\prime}\left(x_{3}\right) u_{3}^{+} .
$$

In addition, from the physical point of view (see [3], [14], [16]-[19], and the references therein), when a subsonic flow in an unbounded domain is called stable, it should admit a determined state at infinity. Thus, along each stream line starting from the shock curve, we naturally pose

$$
\lim _{\mathrm{x}_{3} \rightarrow \infty} \text { along stream line }\left(\rho^{+}, U^{+}, u_{3}^{+}, P^{+}\right) \text {exists for } b\left(x_{3}\right) \leq r \leq \chi\left(x_{3}\right) .
$$

The main result in our paper can be stated as follows.

THEOREM 1.1 (Instability of a global transonic shock). Under the assumptions above, there exists an $\varepsilon_{0}>0$ such that for $\varepsilon<\varepsilon_{0}$, the problem (1.5) with (1.6)-(1.9) has no global solution $\left(\rho^{+}(x), U^{+}(x), u_{3}^{+}(x), P^{+}(x)\right.$; $\left.\chi\left(x_{3}\right)\right)$ which admits the following properties:

(i) $\chi\left(x_{3}\right) \in C^{2}(0, \infty) \cap \operatorname{Lip}[0, \infty)$, and

$$
\left\|\chi^{\prime}\left(x_{3}\right)-s_{0}\right\|_{L^{\infty}(0, \infty)} \leq C\left(\varepsilon_{0}\right)
$$

where below the generic function $C\left(\varepsilon_{0}\right)$ is a suitably small quantity, which is independent of $\varepsilon$.

(ii) $\left(\rho^{+}, U^{+}, u_{3}^{+}, P^{+}\right) \in C^{1}\left(\bar{\Omega}_{+} \backslash(0,0)\right) \cap L^{\infty}\left(\Omega_{+}\right)$, and

$$
\left\|\left(\rho^{+}, U^{+}, u_{3}^{+}, P^{+}\right)\left(x_{3}, r\right)-\left(\hat{\rho}, \hat{U}, \hat{u}_{3}, \hat{P}\right)\left(\frac{r}{x_{3}}\right)\right\|_{L^{\infty}\left(\Omega_{+}\right)} \leq C\left(\varepsilon_{0}\right) .
$$

In addition, if we denote by the domain $\Omega_{+, \delta}=\left\{\left(x_{3}, r\right): x_{3}>\delta, b\left(x_{3}\right)<\right.$ $\left.r<\chi\left(x_{3}\right)\right\}$ with $\delta>0$ any fixed constant, then there exists a quantity $C_{\delta}\left(\varepsilon_{0}\right)>$ 0 depending only on $\delta$ and $\varepsilon_{0}$ such that

$$
\left\|\left(\rho^{+}, U^{+}, u_{3}^{+}, P^{+}\right)\left(x_{3}, r\right)-\left(\hat{\rho}, \hat{U}, \hat{u}_{3}, \hat{P}\right)\left(\frac{r}{x_{3}}\right)\right\|_{C^{1}\left(\Omega_{+, \delta}\right)} \leq C_{\delta}\left(\varepsilon_{0}\right) .
$$


Here $\left(\hat{\rho}\left(r / x_{3}\right), \hat{U}\left(r / x_{3}\right), \hat{u}_{3}\left(r / x_{3}\right), \hat{P}\left(r / x_{3}\right)\right)$ stands for the extension of the self-similar downstream subsonic state $\left(\tilde{\rho}\left(r / x_{3}\right), \tilde{U}\left(r / x_{3}\right), \tilde{u}_{3}\left(r / x_{3}\right), \tilde{P}\left(r / x_{3}\right)\right)$ behind the transonic shock $r=s_{0} x_{3}$, which is formed by the supersonic incoming flow $\left(\rho_{0}, 0,0, q_{0}, P_{0}\right)$ past the cone $\left\{x: \sqrt{x_{1}^{2}+x_{2}^{2}}<b_{0} x_{3}\right\}$. For more detailed information, see Lemma 2.1, Lemma 2.2, and Remark 2.1 in Section 2.

(iii) Denoting by the stream line equation $r=r\left(x_{3}, y\right)$, which starts from the point $(y, \chi(y))$ of the shock with $y>0$, and setting $\lim _{x_{3} \rightarrow \infty}\left(\rho^{+}, U^{+}, u_{3}^{+}\right.$, $\left.P^{+}\right)\left(x_{3}, r\left(x_{3}, y\right)\right)=\left(\rho_{\infty}(y), U_{\infty}(y), u_{3, \infty}(y), P_{\infty}(y)\right)$, then $\left(\rho_{\infty}(y), U_{\infty}(y)\right.$, $\left.u_{3, \infty}(y), P_{\infty}(y)\right) \in C^{1}(0, \infty)$ and

$$
\begin{array}{r}
\lim _{x_{3} \rightarrow \infty} \nabla_{x_{3}, y}\left(\left(\rho^{+}, U^{+}, u_{3}^{+}, P^{+}\right)\left(x_{3}, r\left(x_{3}, y\right)\right)\right. \\
\left.-\left(\rho_{\infty}(y), U_{\infty}(y), u_{3, \infty}(y), P_{\infty}(y)\right)\right)=0 .
\end{array}
$$

REMARK 1.1. With respect to the background solution with the selfsimilar downstream subsonic state $\left(\tilde{\rho}\left(r / x_{3}\right), \tilde{U}\left(r / x_{3}\right), \tilde{u}_{3}\left(r / x_{3}\right), \tilde{P}\left(r / x_{3}\right)\right)$ behind the transonic shock $r=s_{0} x_{3}$, we can show that all the assumptions in Theorem 1.1 hold true. This is given in the Appendix.

REMARK 1.2. For the three-dimensional potential flow equation and related transonic conic shock, the corresponding regularities and estimates of $\left(\rho^{+}, U^{+}, u_{3}^{+}, P^{+}\right)$as stated in Theorem 1.1 have been shown by us in [16] and [17].

REMARK 1.3. Since we have shown in [18] that the limit of the Euler flow along the stream line at infinity does exist, all the analogous estimates to (1.10)-(1.13) of Theorem 1.1 hold for the transonic shock problem on the two-dimensional Euler supersonic flow past a sharp wedge. On the other hand, assumptions (1.10)-(1.13) are fundamental in order to illustrate the global stability of one transonic conic shock.

Remark 1.4. When $\left\|\left(\rho_{\infty}(y), U_{\infty}(y), u_{3, \infty}(y), P_{\infty}(y)\right)\right\|_{C^{1, \delta}(0, \infty)} \leq C$ with some constant $0<\delta<1$, then (1.13) in Theorem 1.1(iii) can be derived from

$$
\lim _{x_{3} \rightarrow \infty}\left(\rho^{+}, U^{+}, u_{3}^{+}, P^{+}\right)\left(x_{3}, r\left(x_{3}, y\right)\right)=\left(\rho_{\infty}(y), U_{\infty}(y), u_{3, \infty}(y), P_{\infty}(y)\right)
$$

and (1.12) of Theorem 1.1 by use of the interpolation formula.

We note that there have been many studies on the transonic problems (e.g., [1], [3], [11], [12], [13] and references therein). In particular, we mention some works which are related to this paper. As illustrated in [6], if a uni- 
form supersonic flow $\left(\rho_{0}, 0,0, q_{0}, P_{0}\right)$ comes from minus infinity, and the flow hits the sharp circular cone $\sqrt{x_{1}^{2}+x_{2}^{2}}=b_{0} x_{3}$ along the axis $x_{3}$-direction, it follows from the Rankine-Hugoniot conditions and the entropy condition that there will appear a weak or a strong self-similar shock attached at the vertex of the cone. With respect to the weak shocks, under some different assumptions, the authors in [4], [5], [7], [8], and [15] have established the local or global existence and stability for the perturbed supersonic incoming flow past a sharp cone when the pressure of downstream region at infinity is appropriately smaller than that of the incoming flow. With respect to transonic shocks, for the symmetrically or multidimensionally perturbed supersonic incoming flow and the potential equation, we have shown the global existence and stability of a steady transonic shock wave solution in [16] and [17], respectively. Since the full Euler system is a more precise model to describe the transonic shock problem (in the general case, the potential flow equation can be used to study the weak shock problem with physical sense), in this paper we focus on the global transonic shock wave problem for the supersonic Euler flow past a sharp cone. Surprisingly, because of the crucial influence of the rotation in the Euler system, it is shown that the transonic shock is unstable. More concretely, we prove the instability of a global transonic conic shock wave, which is similar to the well-known nonexistence conclusions in [14], where it is shown that the continuous transonic flows past profiles are unstable and do not exist in general if the surfaces of the profiles are arbitrarily perturbed.

In order to prove Theorem 1.1, our key observation is that the transonic shock curve must be straight if the transonic shock solution is assumed to be globally stable in the downstream subsonic region, due to the essential influence of the rotation in the Euler system (see (3.12) and (3.18) in $\S 3$ below). Subsequently, we can derive that the subsonic solution will be analytic in the subsonic region by use of the analytic theory of second-order nonlinear elliptic equations with analytic coefficients (see, e.g., [2] and [9]). From this, it is shown that the perturbed conic surface must be the same as $r=b_{0} x_{3}$. Obviously, this is a contradiction. Consequently, we complete the proof on Theorem 1.1.

Our paper is organized as follows. In $\S 2$, for the full Euler system, we show that there exists an attached transonic conic shock $r=s_{0} x_{3}$ for suitable $b_{0}>0$ when a uniform supersonic incoming flow $\left(\rho_{0}, 0,0, q_{0}, P_{0}\right)$ hits 
the cone $r=b_{0} x_{3}$. This is achieved by the shooting method on the nonlinear ordinary differential system with two-point boundary values, where one boundary is free. In $\S 3$, we show that the transonic shock curve must be straight under the assumptions of Theorem 1.1. In $\S 4$, by use of the analytic theory of solutions to second-order nonlinear elliptic equations with analytic coefficients, we show that the subsonic solution is analytic in the downstream region and, further, derive that the perturbed conic surface $r=b\left(x_{3}\right)$ is only $r=b_{0} x_{3}$. From this contradiction, Theorem 1.1 can be proved. In the Appendix, we give the proof for Remark 1.1.

\section{$\S 2$. Self-similar transonic shock solution and its properties}

In R. Courant and K. O. Friedrichs [6], the following transonic conic shock phenomena for the supersonic flow past a sharp cone are illustrated: Suppose that there is a uniform supersonic flow $\left(\rho_{0}, 0,0, q_{0}, P_{0}\right)$ and that the flow hits the circular cone along the $x_{3}$-direction. The conic surface is described by $r=b_{0} x_{3}$; then there exists a critical value $b^{*}$ such that there will appear a transonic conic shock $r=s_{0} x_{3}\left(s_{0}>b_{0}\right)$ attached at the tip for $b_{0}<b^{*}$. Moreover, the solution of (1.5) is self-similar; that is, under the cylindrical coordinates $\left(x_{3}, r\right)$, the solution of (1.5) between the shock front and the conic surface has the form $\rho^{+}(x)=\tilde{\rho}(s), u_{1}^{+}(x)=\tilde{U}(s)\left(x_{1} / r\right), u_{2}^{+}(x)=$ $\tilde{U}(s)\left(x_{2} / r\right), u_{3}^{+}(x)=\tilde{u}_{3}(s)$ and $P^{+}(x)=\tilde{P}(s)$ with $s=r / x_{3}$. With respect to the existence and uniqueness of $\left(\tilde{\rho}(s), \tilde{U}(s), \tilde{u}_{3}(s), \tilde{P}(s) ; s_{0}\right)$, in the case of potential flow equation, we have given the detailed proof in [16] following the ideas in $[6, \S 154-\oint 156]$. Next we show the existence for the full Euler system case and the polytropic gas case. Although the proof procedure is somewhat similar to that in [16], for the reader's convenience and its use later on, we give the details here.

It follows from system (1.5) and a direct computation that the self-similar solution $\left(\tilde{\rho}(s), \tilde{U}(s), \tilde{u}_{3}(s), \tilde{P}(s)\right)$ satisfies the following nonlinear ordinary differential system:

$$
\left\{\begin{array}{l}
\tilde{\rho}^{\prime}(s)=-\frac{\tilde{\rho}^{2} \tilde{U}\left(s \tilde{u}_{3}-\tilde{U}\right)}{s\left(\gamma \tilde{P}\left(1+s^{2}\right)-\tilde{\rho}\left(s \tilde{u}_{3}-\tilde{U}\right)^{2}\right)}, \\
\tilde{U}^{\prime}(s)=-\frac{\gamma \tilde{P} \tilde{U}}{s\left(\gamma \tilde{P}\left(1+s^{2}\right)-\tilde{\rho}\left(s \tilde{u}_{3}-\tilde{U}\right)^{2}\right)}, \quad \text { for } b_{0} \leq s \leq s_{0}, \\
\tilde{u}_{3}^{\prime}(s)=\frac{\gamma \tilde{P} \tilde{U}}{\gamma \tilde{P}\left(1+s^{2}\right)-\tilde{\rho}\left(s \tilde{u}_{3}-\tilde{U}\right)^{2}}, \\
\tilde{P}^{\prime}(s)=-\frac{\gamma \tilde{\rho} \tilde{P} \tilde{U}\left(s \tilde{u}_{3}-\tilde{U}\right)}{s\left(\gamma \tilde{P}\left(1+s^{2}\right)-\tilde{\rho}\left(s \tilde{u}_{3}-\tilde{U}\right)^{2}\right)},
\end{array}\right.
$$


where $\tilde{U}^{2}(s)+\tilde{u}_{3}^{2}(s)<c^{2}(s) \equiv \gamma \tilde{P}(s) / \tilde{\rho}(s)$. Moreover, Bernoulli's law holds true:

$$
\frac{1}{2}\left(\tilde{U}^{2}(s)+\tilde{u}_{3}^{2}(s)\right)+\frac{\gamma \tilde{P}(s)}{(\gamma-1) \tilde{\rho}(s)} \equiv C_{0} .
$$

For notational convenience, below we will drop " $\sim$ " in the solution $(\tilde{\rho}(s)$, $\left.\tilde{U}(s), \tilde{u}_{3}(s), \tilde{P}(s)\right)$. Instead, the notation $\left(\rho(s), U(s), u_{3}(s), P(s)\right)$ will be used directly.

According to Lemma 2.2 below, we know that the denominator $\gamma P(1+$ $\left.s^{2}\right)-\rho\left(s u_{3}-U\right)^{2}>0$ in $(2.1)$ holds for $b_{0} \leq s \leq s_{0}$. This means that the system (2.1) makes sense.

On the shock surface $r=s_{0} x_{3}$, because of the Rankine-Hugoniot conditions and physical entropy condition, one has

$$
\left\{\begin{array}{l}
{[\rho U]-s_{0}\left[\rho u_{3}\right]=0} \\
{\left[P+\rho U^{2}\right]-s_{0}\left[\rho U u_{3}\right]=0} \\
{\left[\rho U u_{3}\right]-s_{0}\left[P+\rho u_{3}^{2}\right]=0}
\end{array}\right.
$$

and

$$
P_{0}<P\left(s_{0}\right)
$$

Additionally,

$$
U\left(b_{0}\right)=b_{0} u_{3}\left(b_{0}\right)
$$

With respect to the nonlinear system (2.1) and (2.2) with free boundary value conditions (2.3) and (2.4) and fixed boundary value condition (2.5), we have the following.

Lemma 2.1. For the given $b_{0}>0$, which is less than some critical value $b^{*}$, we can conclude that the problem (2.1)-(2.5) has a smooth solution $\left(\rho(s), U(s), u_{3}(s), P(s)\right)$ and admits a determined shock position $s=s_{0}$ such that $\sqrt{U^{2}(s)+u_{3}^{2}(s)}<c(s)$ holds true for $b_{0} \leq s \leq s_{0}$.

Proof. Set

$$
\begin{aligned}
\rho_{+} & =\lim _{s \rightarrow s_{0}-0} \rho(s), \quad U_{+}=\lim _{s \rightarrow s_{0}-0} U(s), \\
u_{3+} & =\lim _{s \rightarrow s_{0}-0} u_{3}(s), \quad \text { and } \quad P_{+}=\lim _{s \rightarrow s_{0}-0} P(s) .
\end{aligned}
$$


For convenience, let $\sigma=1 / s$; then $(2.1)$ can be rewritten as

$$
\left\{\begin{array}{l}
\rho^{\prime}(\sigma)=\frac{\rho^{2} U\left(u_{3}-\sigma U\right)}{\gamma P\left(1+\sigma^{2}\right)-\rho\left(u_{3}-\sigma U\right)^{2}}, \\
U^{\prime}(\sigma)=\frac{\sigma \gamma P U}{\gamma P\left(1+\sigma^{2}\right)-\rho\left(u_{3}-\sigma U\right)^{2}}, \\
u_{3}^{\prime}(\sigma)=-\frac{\gamma P U}{\gamma P\left(1+\sigma^{2}\right)-\rho\left(u_{3}-\sigma U\right)^{2}}, \\
P^{\prime}(\sigma)=\frac{\gamma \rho P U\left(u_{3}-\sigma U\right)}{\gamma P\left(1+\sigma^{2}\right)-\rho\left(u_{3}-\sigma U\right)^{2}},
\end{array}\right.
$$

where $\sigma_{0}=1 / s_{0}$.

It follows from a direct computation that

$$
\left(\frac{P}{\rho^{\gamma}}\right)^{\prime}(\sigma)=0
$$

This means that

$$
P(\sigma)=A_{+} \rho^{\gamma}(\sigma)
$$

where $A_{+}=P_{+} / \rho_{+}^{\gamma}$.

By the fourth equation in (1.5) and (2.7), we know that $\rho(\sigma), P(\sigma)$, and the sound speed $c(\sigma)$ can be expressed as the smooth functions of $\left(U(\sigma), u_{3}(\sigma)\right)$, respectively. Namely,

$$
\begin{aligned}
\rho(\sigma) & =\rho\left(U(\sigma), u_{3}(\sigma)\right), \quad P(\sigma)=P\left(U(\sigma), u_{3}(\sigma)\right), \quad \text { and } \\
(2.8) \quad c(\sigma) & =c\left(U(\sigma), u_{3}(\sigma)\right) .
\end{aligned}
$$

In addition, by the second and third equation in (2.6), we find that

$$
\frac{U^{\prime}(\sigma)}{u_{3}^{\prime}(\sigma)}=-\sigma
$$

As indicated in $[6, \S 155]$, it is particularly amenable to treat $U$ as a function of $u_{3}$. In this case, we have

$$
U^{\prime}\left(u_{3}\right)=-\sigma
$$

and

$$
u_{3}^{\prime}(\sigma)=-\frac{1}{U^{\prime \prime}\left(u_{3}\right)}, \quad U^{\prime}(\sigma)=-\frac{U^{\prime}\left(u_{3}\right)}{U^{\prime \prime}\left(u_{3}\right)}
$$




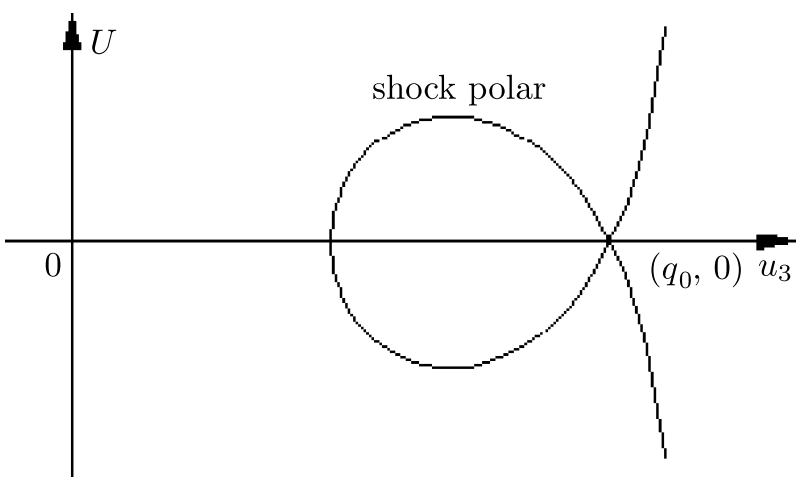

Figure 2

Substituting (2.10) into the second equation of (2.6) and using (2.7) and (2.8) yield

$$
U U^{\prime \prime}\left(u_{3}\right)=1+\left(U^{\prime}\left(u_{3}\right)\right)^{2}-\frac{\left(u_{3}+U U^{\prime}\left(u_{3}\right)\right)^{2}}{c^{2}} .
$$

Assume that the parameter equation of the shock polar is

$$
u_{3}=u_{3}(t), \quad U=U(t)
$$

whose equation describes the relation of $u_{3}$ and $U$ on the $\left(u_{3}, U\right)$-plane in terms of the right-hand conditions (2.3). Its picture is presented as Figure 2 (see also [6, page 313]).

Next, we discuss the existence of solution to (2.11), which starts from any point $M\left(u_{3}\left(t_{0}\right), U\left(t_{0}\right)\right)$ of the shock polar in the subsonic part. In this case, $U>0, u_{3}>0$, and $\sigma>0$ only are considered.

In light of the second and third equalities in (2.3), we can solve the corresponding $\sigma_{0}$, which is denoted by $\sigma_{0}\left(t_{0}\right)=\left(U\left(t_{0}\right)\right) /\left(q_{0}-u_{3}\left(t_{0}\right)\right)$. From $(2.9)$, we have $U_{u_{3}}^{\prime}\left(u_{3}\left(t_{0}\right)\right)=-\sigma_{0}\left(t_{0}\right)$.

Now we study the following initial value problem:

$$
\left\{\begin{array}{l}
c^{2} U U^{\prime \prime}\left(u_{3}\right)=\left(c^{2}-U^{2}\right)\left(U^{\prime}\right)^{2}\left(u_{3}\right)-2 u_{3} U U^{\prime}\left(u_{3}\right)+\left(c^{2}-u_{3}^{2}\right), \\
U\left(u_{3}^{0}\right)=U^{0}, U^{\prime}\left(u_{3}^{0}\right)=-\sigma_{0}
\end{array}\right.
$$

where $u_{3}^{0}=u_{3}\left(t_{0}\right), U^{0}=U\left(t_{0}\right)$, and $\sigma_{0}=\sigma_{0}\left(t_{0}\right)$. From the theory of ODE and $U^{0}>0,(2.12)$ is locally solvable. 


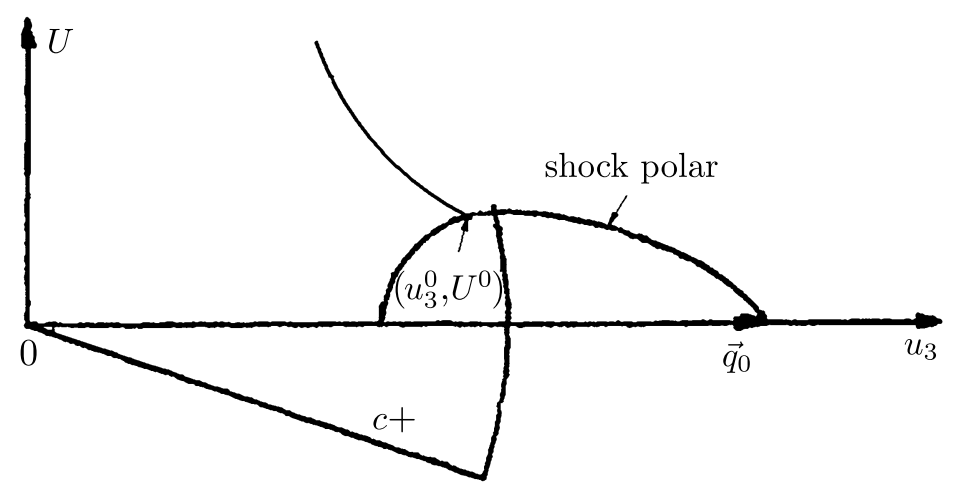

Figure 3

Next, we assert that (2.12) is actually solved in the first quadrant when $\sigma_{0} \leq \sigma \leq 1 / b_{0}$. Indeed, by the discriminant $\Delta=4\left(\left(u_{3} U\right)^{2}-\left(c^{2}-u_{3}^{2}\right)\left(c^{2}-\right.\right.$ $\left.\left.U^{2}\right)\right)=4 c^{2}\left(u_{3}^{2}+U^{2}-c^{2}\right)<0$ and $c^{2}-U^{2}>0$ in the subsonic domain, we then obtain that the term $\left(1 / c^{2}\right)\left(\left(c^{2}-u_{3}^{2}\right)-2 u_{3} U U^{\prime}\left(u_{3}\right)+\left(c^{2}-U^{2}\right)\left(U^{\prime}\left(u_{3}\right)\right)^{2}\right)$ in the right-hand side of $(2.11)$ is greater than 0 . From this we derive that $U^{\prime \prime}\left(u_{3}\right)>0$ holds true. By $(2.10)$, one has $u_{3}^{\prime}(\sigma)<0$ and $U^{\prime}(\sigma)>0$, which means that the solution curve of (2.12) extends from southeast to northwest and that $U^{\prime}\left(u_{3}\right)$ decreases along the solution curve (but $\left|U^{\prime}\left(u_{3}\right)\right|$ increases) (see Figure 3). Since $\sigma \in\left[\sigma_{0}, 1 / b_{0}\right]$, one can then derive that

$$
U-U^{0}=\int_{u_{3}^{0}}^{u_{3}} U_{u_{3}}^{\prime}(z) d z=\int_{u_{3}}^{u_{3}^{0}} \sigma d z \leq \int_{0}^{u_{3}^{0}} \frac{1}{b_{0}} d z \leq \frac{u_{3}^{0}}{b_{0}} .
$$

Thus, $U^{0} \leq U \leq\left(u_{3}^{0} / b_{0}\right)+U^{0}$ holds true. Together with $\left|U_{u_{3}}^{\prime}\right| \leq 1 / b_{0}$, it follows from the extension theorem of the solution to the ODE that (2.12) is always solvable in the subsonic domain of the first quadrant. On the other hand, it follows from (2.1) and Lemma 2.2(i) below that $\left(U^{2}+u_{3}^{2}-c^{2}\right)(\sigma) \leq$ $\left(U^{2}+u_{3}^{2}-c^{2}\right)\left(\sigma_{0}\right)<0$, which means that $(2.12)$ is solved for $\sigma \in\left[\sigma_{0}, 1 / b_{0}\right]$.

Next, for fixed $t_{0}$, we associate $(2.12)$ with the shock boundary condition in (2.3) and fixed wall boundary condition in (2.5). For the requirement of (2.5), we need to look for a point $N$ in the $\left(u_{3}, U\right)$-plane such that the solution curve of (2.12) ends $N$ and fulfills

$$
\left.\left(u_{3}, U\right)\left(1, U_{u_{3}}^{\prime}\right)\right|_{N}=0
$$


which is equivalent to

$$
\arctan \frac{U}{u_{3}}-\arctan U^{\prime}\left(u_{3}\right)=\frac{\pi}{2} \quad \text { at } N .
$$

Now we show that there exists a determined point $N$ such that (2.13) holds. When point $N$ moves along the solution curve of (2.13) (correspondingly, $\sigma$ increases), then $\arctan U / u_{3}$ increases and $\arctan U^{\prime}\left(u_{3}\right)$ decreases. At $\sigma=\sigma_{0}$, from the right-hand condition of (2.3), we know that $U^{0} / u_{3}^{0}<$ $k_{\text {shock }}=1 / \sigma_{0}=\left(q_{0}-u_{3}^{0}\right) / U^{0}$. This derives

$$
\arctan \frac{U^{0}}{u_{3}^{0}}-\arctan U^{\prime}\left(u_{3}^{0}\right)=\arctan \frac{U^{0}}{u_{3}^{0}}+\arctan \frac{U^{0}}{q_{0}-u_{3}^{0}} \leq \frac{\pi}{2},
$$

where the equality holds if and only if $\left(u_{3}^{0}, U^{0}\right)=\left(q_{0}, 0\right)$. Moreover, noting that $U / u_{3} \geq U^{0} / u_{3} \rightarrow+\infty$ and $\arctan U / u_{3} \rightarrow \pi / 2$ as $u_{3} \rightarrow 0,-\arctan U_{u_{3}}^{\prime} \geq$ $\arctan \sigma_{0}$ holds. This yields $\arctan U / u_{3}-\arctan U^{\prime}\left(u_{3}\right)>\pi / 2$ as $u_{3} \rightarrow 0$. Thus, by the continuity and monotone of $\arctan U / u_{3}-\arctan U^{\prime}\left(u_{3}\right)$ with respect to $u_{3}$, there must exist a unique $N$ such that the arc $M N$ corresponds to the solution of (2.11) together with two boundary values $U\left(u_{3}^{0}\right)=$ $U^{0}$ and $\left.\left(u_{3}, U\right)\left(1, U_{u_{3}}^{\prime}\right)\right|_{N}=0$.

Next, we show that for any sharp body, there exists a unique transonic shock such that the boundary value problem (2.12) with (2.3) and (2.5) is always solvable. Indeed, when $M\left(u_{3}\left(t_{0}\right), U\left(t_{0}\right)\right)$ moves at the shock polar in the subsonic domain, it follows from $\left(u_{3}\left(t_{0}\right), U\left(t_{0}\right)\right) \in C^{k}$ and the continuous dependence of solution on the initial values that $\left.\left(u_{3}, U\right)\right|_{N}$ is of $C^{k}$ on $t_{0}$. This continuous curve (together with the supersonic shock part), which is composed of $N$, is called the apple curve, which lies above the shock polar (see Figure 4, from [6]).

If $b_{0} \rightarrow 0$, by use of $\left.U_{u_{3}}^{\prime}\right|_{N}=-1 / b_{0}$, we then have $\left.U_{u_{3}}^{\prime}\right|_{N} \rightarrow-\infty$ and $\left.\arctan U_{u_{3}}^{\prime}\right|_{N} \rightarrow-\pi / 2$. Hence, it follows from (2.13) and $0<u_{3} \leq u_{3}^{0}<\infty$ that $\left.\arctan \left(U / u_{3}\right)\right|_{N} \rightarrow 0$ and $\left.U\right|_{N} \rightarrow 0$. This implies, when $b_{0}$ is less than the critical value $b^{*}$, that the radial line $U=b_{0} u_{3}$ will intersect with the apple curve in the subsonic part. Moreover, by use of (2.13) and the uniqueness theorem of the solution to ODE, we know that the nonlinear mapping from $N$ to $M$, which is determined by (2.12) and (2.13), is one to one between the apple curve and the shock polar. Namely, the subsonic solution of (2.12) exists uniquely when $b_{0}<b^{*}$. This, together with (2.8), yields Lemma 2.1. 


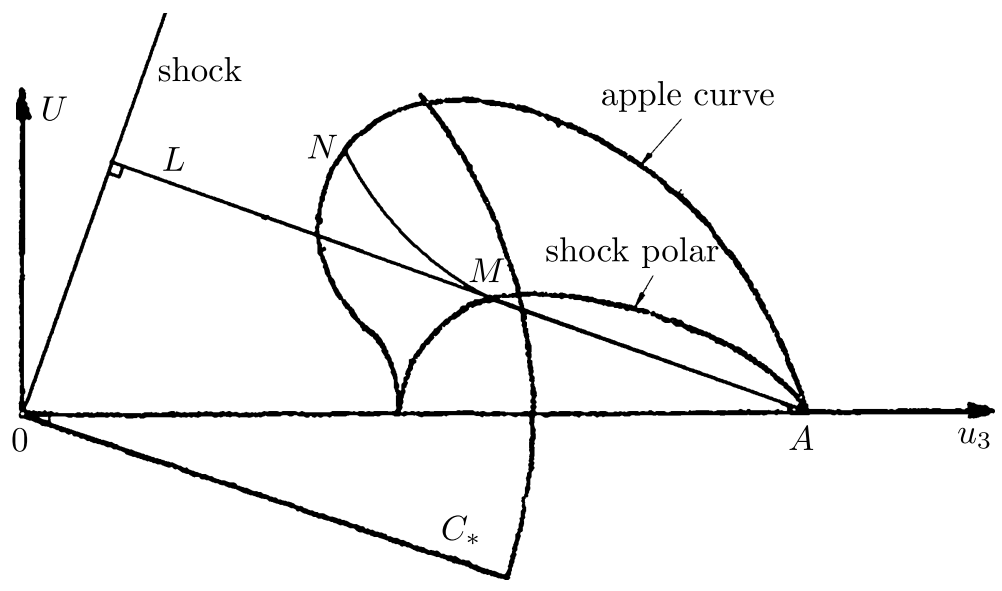

Figure 4

Next, we establish some interesting properties on $\left(\rho(s), U(s), u_{3}(s), P(s)\right)$, which can be used to illustrate that the system (2.1) makes sense.

LEMMA 2.2. If $b_{0}$ is less than a critical value $b^{*}$ and if $\sqrt{U^{2}\left(s_{0}\right)+u_{3}^{2}\left(s_{0}\right)}<$ $c\left(s_{0}\right)$ holds true, then for $b_{0} \leq s \leq s_{0}$, the solution $\left(\rho(s), U(s), u_{3}(s), P(s)\right)$ of (2.1)-(2.5) satisfies the following.

(i) $U^{\prime}(s)<0, u_{3}^{\prime}(s)>0, \rho^{\prime}(s)<0$, and $\left(U^{2}+u_{3}^{2}-c^{2}\right)^{\prime}(s)>0$.

(ii) $U^{2}(s)+u_{3}^{2}(s)<c^{2}(s)$, and $c^{2}(s)\left(1+s^{2}\right)-\left(s u_{3}(s)-U(s)\right)^{2}>0$.

Proof. We denote by $\rho_{+}=\lim _{s \rightarrow s_{0}-0} \rho(s), U_{+}=\lim _{s \rightarrow s_{0}-0} U(s), u_{3+}=$ $\lim _{s \rightarrow s_{0}-0} u_{3}(s)$, and $P_{+}=\lim _{s \rightarrow s_{0}-0} P(s)$.

By the right-hand conditions of (2.3) and Bernoulli's law (2.2), we can compute $u_{3+}$ and $U_{+}$as follows, which can be found in $[6, \S 121]$ :

$$
\left\{\begin{array}{l}
u_{3+}=\left(1-\mu^{2}\right) q_{0} \cos ^{2} \beta+\frac{c_{*}^{2}}{q_{0}} \\
U_{+}=\left(q_{0}-u_{3+}\right) \cot \beta
\end{array}\right.
$$

where $\mu^{2}=(\gamma-1) /(\gamma+1), \beta=\arctan s_{0}$, and $c_{*}^{2}=\mu^{2} q_{0}^{2}+\left(1-\mu^{2}\right)\left(\gamma P_{0} / \rho_{0}\right)$.

Equivalently, we have

$$
\left\{\begin{array}{l}
u_{3+}=\frac{2 q_{0}}{(\gamma+1)\left(1+s_{0}^{2}\right)}+\frac{(\gamma-1) q_{0}}{\gamma+1}+\frac{2 \gamma P_{0}}{(\gamma+1) \rho_{0} q_{0}}, \\
U_{+}=\frac{2 s_{0} q_{0}}{(\gamma+1)\left(1+s_{0}^{2}\right)}-\frac{2 \gamma P_{0}}{(\gamma+1) \rho_{0} q_{0} s_{0}} .
\end{array}\right.
$$


In addition, $U_{+}>0$ holds true because of the physical entropy condition, and this yields, from the second equality of (2.15),

$$
0<\frac{\gamma P_{0}}{\rho_{0}}<\frac{s_{0}^{2} q_{0}^{2}}{1+s_{0}^{2}} .
$$

By use of the Bernoulli law, we arrive at

$$
\frac{\gamma P_{+}}{\rho_{+}}=\frac{\gamma P_{0}}{\rho_{0}}+\frac{\gamma-1}{2}\left(q_{0}^{2}-u_{3+}^{2}-U_{+}^{2}\right) .
$$

It follows from (2.15)-(2.17) and direct computation that

$$
\begin{aligned}
& \frac{\gamma P_{+}}{\rho_{+}}\left(1+s_{0}^{2}\right)-\left(s_{0} u_{3+}-U_{+}\right)^{2} \\
& =\frac{1}{q_{0}^{2} s_{0}^{2}(1+\gamma)}\left(-2\left(1+s_{0}^{2}\right)^{2}\left(\frac{\gamma P_{0}}{\rho_{0}}\right)^{2}\right. \\
& \left.\quad+s_{0}^{2}\left(1+s_{0}^{2}\right) q_{0}^{2}(3-\gamma) \frac{\gamma P_{0}}{\rho_{0}}+q_{0}^{4} s_{0}^{4}(\gamma-1)\right) \\
& =\frac{2\left(1+s_{0}^{2}\right)^{2}}{s_{0}^{2} q_{0}^{2}(1+\gamma)}\left(\frac{s_{0}^{2} q_{0}^{2}}{1+s_{0}^{2}}-\frac{\gamma P_{0}}{\rho_{0}}\right)\left(\frac{\gamma P_{0}}{\rho_{0}}+\frac{(\gamma-1) s_{0}^{2} q_{0}^{2}}{2\left(1+s_{0}^{2}\right)}\right) \\
& >0
\end{aligned}
$$

From (2.8) and the state equation of polytropic gas, we know that the entropy $S$ behind the shock is a constant, which is denoted by $S_{+}$. In this case,

$$
c(s)=c\left(\rho(s), S_{+}\right)=\frac{\gamma P(s)}{\rho(s)} .
$$

By the continuity of $\rho(s), U(s), u_{3}(s)$, and $P(s),(2.15)$ holds true in $s_{0}-$ $\delta \leq s \leq s_{0}$ with some small $\delta>0$, and then (2.1) makes sense in this interval. Because of (2.1), we know that $\rho^{\prime}(s)<0, U^{\prime}(s)<0, u_{3}^{\prime}(s)>0$, and $P^{\prime}(s)<0$ are valid in $s_{0}-\delta \leq s \leq s_{0}$. In addition, we have

$$
\begin{aligned}
\left(c(s)-\frac{s u_{3}(s)-U(s)}{\sqrt{1+s^{2}}}\right)^{\prime}= & c_{\rho}^{\prime}\left(\rho(s), S_{+}\right) \rho^{\prime}(s) \\
& -\frac{s u_{3}^{\prime}(s)-U^{\prime}(s)}{\sqrt{1+s^{2}}}-\frac{u_{3}(s)+s U(s)}{\left(1+s^{2}\right)^{\frac{3}{2}}}<0 .
\end{aligned}
$$


This means that $c(s)-\left(s u_{3}(s)-U(s)\right) / \sqrt{1+s^{2}}$ is a decreasing function of $s$. Thus, we can conclude from $s_{0}-\delta \leq s \leq s_{0}$ that $U(s) \geq U_{+}$and that $\rho(s) \geq \rho_{+}$, and in terms of (2.18), that

$$
\begin{aligned}
& \frac{\gamma P(s)}{\rho(s)}\left(1+s^{2}\right)-\left(s u_{3}(s)-U(s)\right)^{2} \\
& \quad=c^{2}(s)\left(1+s^{2}\right)-\left(s u_{3}(s)-U(s)\right)^{2} \\
& \quad=\left(1+s^{2}\right)\left(c(s)-\frac{s u_{3}(s)-U(s)}{\sqrt{1+s^{2}}}\right)\left(c(s)+\frac{s u_{3}(s)-U(s)}{\sqrt{1+s^{2}}}\right) \\
& \quad \geq c\left(s_{0}\right)\left(1+b_{0}^{2}\right)\left(c\left(s_{0}\right)-\frac{s_{0} u_{3+}-U_{+}}{\sqrt{1+s_{0}^{2}}}\right)>0
\end{aligned}
$$

From (2.19), one can derive that the denominator in (2.1) is lower bounded away from zero as long as the solution of (2.1) exists. Therefore, (2.19) holds in the whole interval $\left[b_{0}, s_{0}\right]$; meanwhile, the solution of $(2.1)$ exists uniquely by the proof procedure of Lemma 2.1, which satisfies

$$
\rho^{\prime}(s)<0, \quad U^{\prime}(s)<0, \quad u_{3}^{\prime}(s)>0, \quad P^{\prime}(s)<0 .
$$

Moreover, by a direct computation, we have

$$
\left\{\begin{array}{l}
\left(s u_{3}(s)-U(s)\right)^{\prime}=s u_{3}^{\prime}(s)+u_{3}(s)-U^{\prime}(s)>0 \\
\left(u_{3}^{2}(s)+U^{2}(s)-c^{2}(s)\right)^{\prime}=\frac{2\left(\rho c_{\rho}^{\prime}\left(\rho(s), S_{+}\right)+c(s)\right) c(s)\left(s u_{3}-U\right) U}{s\left(\left(1+s^{2}\right) c^{2}(s)-\left(s u_{3}-U\right)^{2}\right)}>0 .
\end{array}\right.
$$

This obviously yields

$$
\begin{gathered}
s u_{3}(s)-U(s) \geq b_{0} u_{3}\left(b_{0}\right)-U\left(b_{0}\right)=0, \\
u_{3}^{2}(s)+U^{2}(s)-c^{2}(s) \leq u_{3}^{2}\left(s_{0}\right)+U^{2}\left(s_{0}\right)-c^{2}\left(s_{0}\right)<0 .
\end{gathered}
$$

Namely, the problem $(2.1)-(2.5)$ has a subsonic solution if $u_{3}^{2}\left(s_{0}\right)+U^{2}\left(s_{0}\right)-$ $c^{2}\left(s_{0}\right)<0$. We thus complete the proof on Lemma 2.2 .

REMARK 2.1. Since the denominator of the system (2.1) is positive in $\left[b_{0}, s_{0}\right]$, by use of the system (2.1) we can extend the background solution $\left(\tilde{\rho}(s), \tilde{U}(s), \tilde{u}_{3}(s), \tilde{P}(s)\right)$ of $(2.1)-(2.5)$ to some interval $\left[b_{0}, s_{0}+\tau_{0}\right]$ with $\tau_{0}>$ 0 . The related extensions are denoted by $\left(\hat{\rho}(s), \hat{U}(s), \hat{u}_{3}(s), \hat{P}(s)\right)$. 


\section{$\S 3$. Some properties on globally stable transonic conic shock waves of a full Euler system}

In this section, under the assumptions of Theorem 1.1, we focus on some basic observations on the globally stable transonic conic wave solutions for the full Euler system.

It is noted that it follows from the first equation of (1.5) that one can introduce a stream function $\psi\left(x_{3}, r\right)$ with $\psi(0,0)=0$ such that $\partial_{x_{3}} \psi\left(x_{3}, r\right)=$ $-r \rho^{+} U^{+}$and $\partial_{r} \psi\left(x_{3}, r\right)=r \rho^{+} u_{3}^{+}$.

In addition, by (1.5) and the state equation, we can easily deduce $\left(U^{+} \partial_{r}+\right.$ $\left.u_{3}^{+} \partial_{x_{3}}\right)\left(P^{+} /\left(\rho^{+}\right)^{\gamma}\right)=0$. This means that $P^{+} /\left(\rho^{+}\right)^{\gamma}=$ constant holds true along each stream line, which is denoted by a function $Q$. Moreover, it can be shown that the function $Q$ depends only on the stream function $\psi$; that is, $Q$ is expressed as

$$
Q=Q(\psi)
$$

We now prove this assertion.

Let $r=r\left(x_{3}, h\right)$ be the stream line starting from the point $(0, h)$; then it satisfies

$$
\left\{\begin{array}{l}
\frac{d r\left(x_{3}, h\right)}{d x_{3}}=\left(\frac{U}{u_{3}}\right)\left(x_{3}, r\left(x_{3}, h\right)\right), \\
r(0, h)=h ;
\end{array}\right.
$$

here we point out that the function $r\left(x_{3}, h\right)$ is $C^{\infty}$ in the supersonic region (in fact, $r\left(x_{3}, h\right) \equiv h$ ), $C^{2}$ in the subsonic region, and Lipschitzian across the shock. On the other hand, because of $\left(\rho^{+}, U^{+}, u_{3}^{+}, P^{+}\right) \in C^{1}\left(\bar{\Omega}_{+} \backslash(0,0)\right) \cap$ $L^{\infty}\left(\Omega_{+}\right)$and the assumptions (1.11) and (1.12), we know that $\left(U / u_{3}\right)\left(x_{3}\right.$, $\left.r\left(x_{3}, h\right)\right) \in C^{1}\left(\Omega_{+}\right)$and has a positive lower bound by the uniform positive lower bound of the background solution $\left(\hat{\rho}, \hat{U}, \hat{u}_{3}, \hat{P}\right)\left(r / x_{3}\right)$ and the smallness of $C\left(\varepsilon_{0}\right)$ in Theorem 1.1. Thus, (3.2) has a global solution, and $r\left(x_{3}, h\right) \rightarrow+\infty$ as $x_{3} \rightarrow+\infty$.

Since

$$
\begin{aligned}
\partial_{x_{3}} & \int_{r\left(x_{3}, 0\right)}^{r\left(x_{3}, h\right)} r \rho u_{3} d r \\
= & r \rho u_{3}\left(x_{3}, r\left(x_{3}, h\right)\right) \frac{d r\left(x_{3}, h\right)}{d x_{3}}-r \rho u_{3}\left(x_{3}, r\left(x_{3}, 0\right)\right) \frac{d r\left(x_{3}, 0\right)}{d x_{3}} \\
& \quad+\int_{r\left(x_{3}, 0\right)}^{r\left(x_{3}, h\right)} \partial_{x_{3}}\left(r \rho u_{3}\right) d r
\end{aligned}
$$




$$
\begin{aligned}
& =r \rho U\left(x_{3}, r\left(x_{3}, h\right)\right)-r \rho U\left(x_{3}, r\left(x_{3}, 0\right)\right)-\int_{r\left(x_{3}, 0\right)}^{r\left(x_{3}, h\right)} \partial_{r}(r \rho U) d r \\
& =0
\end{aligned}
$$

this means that $\int_{r\left(x_{3}, 0\right)}^{r\left(x_{3}, h\right)} r \rho u_{3} d r$ is independent of $x_{3}$. Set $\eta=\int_{r\left(x_{3}, 0\right)}^{r\left(x_{3}, h\right)} r \rho u_{3} d r$; then one can define a well-defined function $h=h(\eta)$.

Let us introduce the Lagrange coordinate $(\xi, \eta)$ as follows:

$$
x_{3}=\xi, r=r(\xi, h(\eta))
$$

It is noted that

$$
\operatorname{det}\left(\frac{\partial\left(x_{3}, r\right)}{\partial(\xi, \eta)}\right)=\partial_{\eta}(r(\xi, h(\eta)))=\frac{1}{r(\xi, h(\eta))\left(\rho^{+} u_{3}^{+}\right)\left(x_{3}, r(\xi, h(\eta))\right)}>0
$$

holds true in $\Omega_{+}=\left\{\left(x_{3}, r\right): x_{3}>0, b\left(x_{3}\right)<r<\chi\left(x_{3}\right)\right\}$ because of the uniform positive lower bound of $\left(\rho^{+}, U^{+}, u_{3}^{+}, P^{+}\right)$. Namely, (3.3) is invertible.

Under the Lagrange transformation (3.3), the system (1.5) has a form

$$
\left\{\begin{array}{l}
\partial_{\xi}\left(\frac{1}{r \rho^{+} u_{3}^{+}}\right)-\partial_{\eta}\left(\frac{U^{+}}{u_{3}^{+}}\right)=0, \\
\partial_{\xi} U^{+}+r \partial_{\eta} P^{+}=0 \\
\rho^{+} u_{3}^{+} \partial_{\xi} u_{3}^{+}+\partial_{\xi} P^{+}-r \rho^{+} U^{+} \partial_{\eta} P^{+}=0 \\
\frac{1}{2}\left(\left(U^{+}\right)^{2}+\left(u_{3}^{+}\right)^{2}\right)+\frac{\gamma P^{+}}{(\gamma-1) \rho^{+}}=C_{0} .
\end{array}\right.
$$

On the other hand, (3.4) can be rewritten as

$$
\left\{\begin{array}{l}
\partial_{\xi}\left(\frac{1}{r \rho^{+} u_{3}^{+}}\right)-\partial_{\eta}\left(\frac{U^{+}}{u_{3}^{+}}\right)=0, \\
\partial_{\xi} U^{+}+r \partial_{\eta} P^{+}=0 \\
\partial_{\xi}\left(\frac{P^{+}}{r \rho^{+} u_{3}^{+}}\right)-\partial_{\eta}\left(\frac{P^{+} U^{+}}{u_{3}^{+}}\right)+\frac{1}{r} \partial_{\xi} u_{3}^{+}=0 \\
\frac{1}{2}\left(\left(U^{+}\right)^{2}+\left(u_{3}^{+}\right)^{2}\right)+\frac{\gamma P^{+}}{(\gamma-1) \rho^{+}}=C_{0} .
\end{array}\right.
$$

Substituting the second equation of (3.4) into the third equation of (3.4) yields

$$
\frac{\partial_{\xi} P^{+}}{\rho^{+}}+U^{+} \partial_{\xi} U^{+}+u_{3}^{+} \partial_{\xi} u_{3}^{+}=0
$$

Meanwhile, taking the first-order derivative on $\xi$ in the fourth equation of (3.4), one has

$$
U^{+} \partial_{\xi} U^{+}+u_{3}^{+} \partial_{\xi} u_{3}^{+}+\frac{\gamma \partial_{\xi} P^{+}}{(\gamma-1) \rho^{+}}-\frac{\gamma P^{+} \partial_{\xi} \rho^{+}}{(\gamma-1)\left(\rho^{+}\right)^{2}}=0
$$


This, together with (3.6) and (3.7), yields

$$
\partial_{\xi}\left(\ln \frac{P^{+}}{\left(\rho^{+}\right)^{\gamma}}\right)=0 .
$$

Since $\partial_{x_{3}} \eta\left(x_{3}, r\right)=\partial_{x_{3}} \psi\left(x_{3}, r\right), \partial_{r} \eta\left(x_{3}, r\right)=\partial_{r} \psi\left(x_{3}, r\right)$, and $\eta(0,0)=$ $\psi(0,0)=0$ hold true, then $\eta\left(x_{3}, r\right) \equiv \psi\left(x_{3}, r\right)$ and

$$
\frac{P^{+}}{\left(\rho^{+}\right)^{\gamma}}=Q\left(\psi\left(x_{3}, r\right)\right) ;
$$

hence, (3.1) is proved.

Next, we derive a basic relation between $Q(\psi)$ and the rotation $\partial_{x_{3}} U^{+}-$ $\partial_{r} u_{3}^{+}$. Substituting the first equation of (1.5) and the expression $P^{+}=$ $Q\left(\psi\left(x_{3}, r\right)\right)\left(\rho^{+}\right)^{\gamma}$ into the second and the third equations in (1.5) yields, respectively,

$$
\left\{\begin{array}{l}
\partial_{r}\left(r \rho^{+} U^{+}\right)+\partial_{x_{3}}\left(r \rho^{+} u_{3}^{+}\right)=0, \\
\rho^{+} U^{+} \partial_{r} U^{+}+\rho^{+} u_{3}^{+} \partial_{3} U^{+}+\partial_{r} P^{+}=0 \\
\rho^{+} U^{+} \partial_{r} u_{3}^{+}+\rho^{+} u_{3}^{+} \partial_{x_{3}} u_{3}^{+}+\partial_{x_{3}} P^{+}=0
\end{array}\right.
$$

and

$$
\left\{\begin{array}{l}
\partial_{r}\left(r \rho^{+} U^{+}\right)+\partial_{x_{3}}\left(r \rho^{+} u_{3}^{+}\right)=0 \\
U^{+} \partial_{r} U^{+}+u_{3}^{+} \partial_{3} U^{+}+Q^{\prime}(\psi)\left(\rho^{+}\right)^{\gamma-1} \partial_{r} \psi \\
\quad+\gamma Q(\psi)\left(\rho^{+}\right)^{\gamma-2} \partial_{r} \rho^{+}=0 \\
U^{+} \partial_{r} u_{3}^{+}+u_{3}^{+} \partial_{x_{3}} u_{3}^{+}+Q^{\prime}(\psi)\left(\rho^{+}\right)^{\gamma-1} \partial_{x_{3}} \psi \\
\quad+\gamma Q(\psi)\left(\rho^{+}\right)^{\gamma-2} \partial_{x_{3}} \rho^{+}=0 .
\end{array}\right.
$$

In addition, differentiating the fourth equation of (3.5) with respect to $r$, we have

$$
U^{+} \partial_{r} U^{+}+u_{3}^{+} \partial_{r} u_{3}^{+}+\gamma Q(\psi)\left(\rho^{+}\right)^{\gamma-2} \partial_{r} \rho^{+}+\gamma\left(\rho^{+}\right)^{\gamma-1} Q^{\prime}(\psi) \partial_{r} \psi=0 .
$$

This, together with the second equation in (3.11), yields

$$
Q^{\prime}(\psi)=\frac{(\gamma-1)\left(\partial_{x_{3}} U^{+}-\partial_{r} u_{3}^{+}\right)}{r\left(\rho^{+}\right)^{\gamma}} .
$$

For the stream line $r=r\left(x_{3}, h\right)$ defined in $(3.2)$, since it will intersect with the shock curve $r=\chi\left(x_{3}\right)$ at some certain point $\left(x_{3}^{0}(h), \chi\left(x_{3}^{0}(h)\right)\right)$, it then 
follows from $\psi\left(x_{3}, r\left(x_{3}, h\right)\right) \equiv \psi\left(x_{3}^{0}(h), \chi\left(x_{3}^{0}(h)\right)\right),(3.12)$, assumption (1.11) in Theorem 1.1 (which means that $\rho^{+}$has a uniform positive lower bound for suitably small $\left.C\left(\varepsilon_{0}\right)\right)$, and the property of $r\left(x_{3}, h\right) \rightarrow+\infty$ as $x_{3} \rightarrow+\infty$ that

$$
\lim _{x_{3} \rightarrow \infty}\left(\partial_{x_{3}} U^{+}-\partial_{r} u_{3}^{+}\right)\left(x_{3}, r\left(x_{3}, h\right)\right)=\infty \quad \text { if } Q^{\prime}\left(\psi\left(x_{3}^{0}, \chi\left(x_{3}^{0}\right)\right)\right) \neq 0 .
$$

However, this case is contradictory with assumption Theorem 1.1(ii). Therefore, in order to guarantee the global stability of the transonic shock solution, together with the arbitrariness of $\left(x_{3}^{0}, \chi\left(x_{3}^{0}\right)\right)$ at the shock curve, it then is required to let $\left(\partial_{x_{3}} U^{+}-\partial_{r} u_{3}^{+}\right)\left(x_{3}, \chi\left(x_{3}\right)\right) \equiv 0$ for $x_{3}>0$. From this, we can further derive that the shock equation $r=\chi\left(x_{3}\right)$ is straight. More precisely, we have the following.

LEMma 3.1. Under the assumptions of Theorem 1.1, for $x_{3}>0,\left(\partial_{x_{3}} U^{+}-\right.$ $\left.\partial_{r} u_{3}^{+}\right)\left(x_{3}, \chi\left(x_{3}\right)\right) \equiv 0$ holds true if and only if $\chi^{\prime \prime}\left(x_{3}\right) \equiv 0$.

REMARK 3.1. By Lemma 3.1, it is easy to see that $\left(\partial_{x_{3}} U^{+}-\partial_{r} u_{3}^{+}\right)\left(x_{3}\right.$, $\left.\chi\left(x_{3}\right)\right) \equiv 0$ holds true for $x_{3}>0$ if and only if $\chi\left(x_{3}\right)=\tilde{s}_{0} x_{3}$ because of $\chi(0)=0$ and the assumption $\chi\left(x_{3}\right) \in C^{2}(0, \infty) \cap \operatorname{Lip}[0, \infty)$ in Theorem 1.1, where $\tilde{s}_{0}$ is some suitable positive constant.

Proof. It follows from (1.6) that on $r=\chi\left(x_{3}\right)$,

$$
\left\{\begin{array}{l}
\rho^{+} U^{+}-\rho^{+} u_{3}^{+} \chi^{\prime}=-\rho_{0} q_{0} \chi^{\prime} \\
P^{+}+\rho^{+}\left(U^{+}\right)^{2}-\chi^{\prime} \rho^{+} U^{+} u_{3}^{+}=P_{0} \\
\rho^{+} U^{+} u_{3}^{+}-P^{+} \chi^{\prime}-\rho^{+}\left(u_{3}^{+}\right)^{2} \chi^{\prime}=-P_{0} \chi^{\prime}\left(x_{3}\right)-\rho_{0} q_{0}^{2} \chi^{\prime}
\end{array}\right.
$$

Multiplying $\chi^{\prime}$ on two sides of the second equation in (3.13) and then adding the third equation in (3.13) yield

$$
\left(U^{+} \chi^{\prime}+u_{3}^{+}\right)\left(\rho^{+} U^{+}-\rho^{+} u_{3}^{+} \chi^{\prime}\right)=-\rho_{0} q_{0}^{2} \chi^{\prime} .
$$

From this, together with the first equation in (3.13) and the fact that $\chi^{\prime} \neq 0$, we obtain, on $r=\chi\left(x_{3}\right)$,

$$
U^{+} \chi^{\prime}+u_{3}^{+}=q_{0}
$$

Taking $\partial_{x_{3}}$ on (3.14) yields

$$
\chi^{\prime} \partial_{x_{3}} U^{+}+U^{+} \chi^{\prime \prime}+\partial_{x_{3}} u_{3}^{+}+\left(\chi^{\prime}\right)^{2} \partial_{r} U^{+}+\partial_{r} u_{3}^{+} \chi^{\prime}=0 \quad \text { on } r=\chi\left(x_{3}\right)
$$


In addition, by differentiating the second equation of (3.13) with respect to the variable $x_{3}$, we arrive at

$$
\begin{gathered}
\partial_{x_{3}} P^{+}+\chi^{\prime} \partial_{r} P^{+}+\partial_{x_{3}}\left(U^{+}\left(\rho^{+} U^{+}-\rho^{+} u_{3}^{+} \chi^{\prime}\right)\right) \\
+\chi^{\prime} \partial_{r}\left(U^{+}\left(\rho^{+} U^{+}-\rho^{+} u_{3}^{+} \chi^{\prime}\right)\right)=0 .
\end{gathered}
$$

It follows from the second equation, the third equation of (3.13), and (3.16) that

$$
\begin{aligned}
& -\rho^{+} U^{+} \partial_{r} u_{3}^{+}-\rho^{+} u_{3}^{+} \partial_{x_{3}} u_{3}^{+}-\rho^{+} u_{3}^{+}\left(\chi^{\prime}\right)^{2} \partial_{r} U^{+}+\partial_{x_{3}} U^{+}\left(\rho^{+} U^{+}-2 \rho^{+} u_{3}^{+} \chi^{\prime}\right) \\
& +U^{+} \frac{d}{d x_{3}}\left(\rho^{+} U^{+}-\rho^{+} u_{3}^{+} \chi^{\prime}\right)=0 .
\end{aligned}
$$

This, together with the first equation in (3.13), yields

$$
\begin{gathered}
-\rho^{+} U^{+} \partial_{r} u_{3}^{+}-\rho^{+} u_{3}^{+} \partial_{x_{3}} u_{3}^{+}-\rho^{+} u_{3}^{+}\left(\chi^{\prime}\right)^{2} \partial_{r} U^{+} \\
+\partial_{x_{3}} U^{+}\left(\rho^{+} U^{+}-2 \rho^{+} u_{3}^{+} \chi^{\prime}\right)=\rho_{0} q_{0} U^{+} \chi^{\prime \prime} .
\end{gathered}
$$

Combining (3.15) with (3.17), we have

$$
\left(\rho^{+} U^{+}-\rho^{+} u_{3}^{+} \chi^{\prime}\right)\left(\partial_{x_{3}} U^{+}-\partial_{r} u_{3}^{+}\right)=\left(\rho_{0} q_{0}-\rho^{+} u_{3}^{+}\right) U^{+} \chi^{\prime \prime} .
$$

Thanks to the first equation of (3.13), one has, on $r=\chi\left(x_{3}\right)$,

$$
\left(\partial_{x_{3}} U^{+}-\partial_{r} u_{3}^{+}\right) \rho_{0} q_{0} \chi^{\prime}=\rho^{+}\left(U^{+}\right)^{2} \frac{\chi^{\prime \prime}}{\chi^{\prime}} .
$$

Because of $\chi^{\prime}\left(x_{3}\right) \neq 0$ and $U^{+} \neq 0$, then we have

$$
\partial_{x_{3}} U^{+}-\partial_{r} u_{3}^{+}=0 \Leftrightarrow \chi^{\prime \prime}=0 \quad \text { on } r=\chi\left(x_{3}\right) .
$$

Thus, we complete the proof of Lemma 3.1.

From Remark 3.1 and (3.12), we easily obtain the following.

LEMMA 3.2. If the shock is not straight, then the global transonic shock solution is unstable.

In the next section, based on Lemma 3.1 and Remark 3.1, we derive a relation which is contradictory with the boundary condition (1.8) and further complete the proof of Theorem 1.1. 


\section{$\S 4$. Proof of Theorem 1.1}

By Lemma 3.1 and Remark 3.1, when the transonic shock is globally stable, we know that the shock curve must be straight, whose slope is denoted by $\tilde{s}_{0}$. Moreover, under the assumptions in Theorem 1.1, one has $\left|\tilde{s}_{0}-s_{0}\right| \leq C\left(\varepsilon_{0}\right)$.

Because of (3.12), we know that $Q^{\prime}(\psi)=\left((\gamma-1)\left(\partial_{x_{3}} U^{+}-\partial_{r} u_{3}^{+}\right)\right) / r\left(\rho^{+}\right)^{\gamma}$ is a constant along each stream line. In addition, it follows from Lemma 3.1 that $\left(\partial_{x_{3}} U^{+}-\partial_{r} u_{3}^{+}\right)\left(x_{3}, \tilde{s}_{0} x_{3}\right) \equiv 0$. Thus one has $Q^{\prime}(\psi) \equiv 0$. This derives from the fact that $Q(\psi) \equiv$ constant holds true behind the shock $r=\tilde{s}_{0} x_{3}$, which is denoted by a positive constant $A$. Therefore, from (3.9), we have

$$
P^{+}\left(x_{3}, r\right)=A\left(\rho^{+}\right)^{\gamma}\left(x_{3}, r\right) \quad \text { and } \quad \partial_{x_{3}} U^{+}-\partial_{r} u_{3}^{+} \equiv 0 .
$$

From this, it is easy to see that the subsonic flow field behind the shock can be described by the potential flow equation. We now set $\partial_{r} \phi=U^{+}$ and $\partial_{x_{3}} \phi=u_{3}^{+}$with $\phi(0,0)=0$. Then it follows from the second and third equations of (3.10) and the state equation of polytropic flow that

$$
\frac{1}{2}|\nabla \phi|^{2}+h\left(\rho^{+}\right)=C_{0},
$$

where $h\left(\rho^{+}\right)=c^{2}\left(\rho^{+}\right) /(\gamma-1)$ is the specific enthalpy.

By use of (4.2) and the implicit function theorem, it is easy to see that the density function $\rho^{+}(x)$ can be expressed as

$$
\rho^{+}=h^{-1}\left(C_{0}-\frac{1}{2}|\nabla \phi|^{2}\right) \equiv H(\nabla \phi) .
$$

Substituting (4.3) into the first equation in (3.10) yields

$$
\begin{aligned}
& \left(c^{2}(\nabla \phi)-\left(\partial_{x_{3}} \phi\right)^{2}\right) \partial_{x_{3}}^{2} \phi-2 \partial_{x_{3}} \phi \partial_{r} \phi \partial_{r x_{3}}^{2} \phi \\
& \quad+\left(c^{2}(\nabla \phi)-\left(\partial_{r} \phi\right)^{2}\right) \partial_{r}^{2} \phi+\frac{c^{2}(\nabla \phi)}{r} \partial_{r} \phi=0
\end{aligned}
$$

where $c(\nabla \phi) \equiv c(H(\nabla \phi))$.

Next, we determine the value of $\phi$ on the shock. On $r=\tilde{s}_{0} x_{3}$, we have

$$
\begin{aligned}
\phi\left(x_{3}, \tilde{s}_{0} x_{3}\right) & =\int_{0}^{x_{3}} \frac{d}{d x_{3}}\left(\phi\left(x_{3}, \tilde{s}_{0} x_{3}\right)\right) d x_{3} \\
& =\int_{0}^{x_{3}}\left(u_{3}\left(\tilde{s}_{0}\right)+U\left(\tilde{s}_{0}\right) \tilde{s}_{0}\right) d x_{3}
\end{aligned}
$$




$$
=\left(u_{3}\left(\tilde{s}_{0}\right)+U\left(\tilde{s}_{0}\right) \tilde{s}_{0}\right) x_{3} \equiv \varphi_{0}\left(x_{3}\right)
$$

and

$$
\begin{aligned}
\partial_{\vec{n}} \phi\left(x_{3}, \tilde{s}_{0} x_{3}\right) & \equiv\left(u_{3}\left(\tilde{s}_{0}\right), U\left(\tilde{s}_{0}\right)\right) \cdot\left(\frac{\tilde{s}_{0}}{\sqrt{1+\tilde{s}_{0}^{2}}},-\frac{1}{\sqrt{1+\tilde{s}_{0}^{2}}}\right) \\
& =\frac{\tilde{s}_{0} u_{3}\left(\tilde{s}_{0}\right)-U\left(\tilde{s}_{0}\right)}{\sqrt{1+\tilde{s}_{0}^{2}}} \equiv \varphi_{1}\left(x_{3}\right) .
\end{aligned}
$$

In addition, on the conic surface $r=b\left(x_{3}\right), \phi$ satisfies

$$
\partial_{r} \phi=b^{\prime}\left(x_{3}\right) \partial_{x_{3}} \phi .
$$

Denote by the domain $\Omega_{1} \equiv\left\{\left(x_{3}, r\right): b\left(x_{3}\right)<r<\tilde{s}_{0} x_{3}, x_{3}>0\right\}$. Then we have the following.

LEMma 4.1. Under the assumptions of Theorem 1.1, the $\operatorname{Lip}\left(\bar{\Omega}_{1}\right) \cap C^{2}\left(\bar{\Omega}_{1} \backslash\right.$ $(0,0))$-regular solution $\phi$ to $(4.4)-(4.7)$ is analytic in the domain $\bar{\Omega}_{1} \backslash\left\{\left(x_{3}\right.\right.$, $\left.r): r=b\left(x_{3}\right), x_{3} \geq 0\right\}$.

Proof. Arbitrarily choosing a point $M_{0}$ in $\bar{\Omega}_{1} \backslash\left\{\left(x_{3}, r\right): r=b\left(x_{3}\right), x_{3} \geq 0\right\}$, there exists two cases: $M_{0} \in \Omega$ and $M_{0} \in\left\{\left(x_{3}, r\right): r=\tilde{s}_{0} x_{3}, x_{3}>0\right\}$.

For the first case, there exists a positive constant $\delta_{0}$ such that the disk $B\left(M_{0}, \delta_{0}\right) \subset \Omega_{1}$. It follows from the classical regularity theory of the solution to the second-order elliptic equation that $\phi\left(x_{3}, r\right) \in C^{\infty}\left(B\left(M_{0}, \delta_{0}\right)\right.$ ) (see [10]). In addition, by the analytic theory on the second elliptic equation with the analytic coefficients (see [2] or [9]), we can derive that $\phi\left(x_{3}, r\right)$ is analytic in $B\left(M_{0}, \delta_{0}\right)$.

Next, we treat the other case, for $M_{0} \in\left\{\left(x_{3}, r\right): r=\tilde{s}_{0} x_{3}, x_{3}>0\right\}$. For convenience, we now take a rotational transformation as follows:

$$
y_{1}=\cos \theta_{0} x_{3}-\sin \theta_{0} r, \quad y_{2}=\sin \theta_{0} x_{3}+\cos \theta_{0} r,
$$

where $\theta_{0}=(\pi / 2)-\arctan \tilde{s}_{0}$.

In this case, the shock curve $r=\tilde{s}_{0} x_{3}$ is changed into $y_{1}=0$, and (4.4)(4.6) can be rewritten as

$$
\left\{\begin{array}{l}
\left(1-\frac{\left(\partial_{y_{1}} \phi\right)^{2}}{c^{2}\left(\nabla_{y} \phi\right)} \partial_{y_{1}}^{2} \phi-\frac{2 \partial_{y_{1}} \phi \partial_{y_{2}} \phi}{c^{2}\left(\nabla_{y} \phi\right.} \partial_{y_{1} y_{2}}^{2} \phi\right. \\
\quad+\left(1-\frac{\left(\partial_{y_{2}} \phi\right)^{2}}{c^{2}\left(\nabla_{y} \phi\right)}\right) \partial_{y_{2}}^{2} \phi+\frac{\cos \theta_{0} \partial_{y_{2}} \phi-\sin \theta_{0} \partial_{y_{1}} \phi}{\cos \theta_{0} y_{2}-\sin \theta_{0} y_{1}}=0 \\
\phi\left(0, y_{2}\right)=\left(u_{3}\left(\tilde{s}_{0}\right)+U\left(\tilde{s}_{0}\right) \tilde{s}_{0}\right) \sin \theta_{0} y_{2} \equiv \tilde{\varphi}_{1}\left(y_{2}\right), \\
\partial_{y_{1}} \phi\left(0, y_{2}\right)=\tilde{s}_{0} u_{3}\left(\tilde{s}_{0}\right)-U\left(\tilde{s}_{0}\right) \equiv \tilde{\varphi}_{2}\left(y_{2}\right)
\end{array}\right.
$$


where $\phi(y) \equiv \phi\left(\cos \theta y_{1}+\sin \theta y_{2},-\sin \theta y_{1}+\cos \theta y_{2}\right)$.

Meanwhile, the domain $\Omega_{1}$ is changed into $\tilde{\Omega}_{1}:\left\{\left(y_{1}, y_{2}\right): 0<y_{1}<\right.$ $\left.\cot \theta_{0} y_{2}-\csc \theta_{0} b\left(\cos \theta_{0} y_{1}+\sin \theta_{0} y_{2}\right)\right\}$.

For the point $M_{0} \in\left\{y_{1}=0\right\}$, because $\left.\left(1-\left(\partial_{y_{1}} \phi\right)^{2} / c^{2}\left(\nabla_{y} \phi\right)\right)\right|_{M_{0}}>0$, it then follows from the Cauchy-Kowalewsky theorem that there exists a positive constant $\delta$ such that the Cauchy problem (4.9) has an analytic solution $\tilde{\phi}(y)$ in $B\left(M_{0}, \delta\right)$.

Set

$$
\hat{\phi}(y)= \begin{cases}\tilde{\phi}(y) & \text { in } B\left(M_{0}, \delta\right) \cap \overline{\mathbb{R}^{2} \backslash \tilde{\Omega}_{1}}, \\ \phi(y) & \text { in } B\left(M_{0}, \delta\right) \cap \overline{\tilde{\Omega}_{1}} .\end{cases}
$$

It can be easily verified that $\hat{\phi}(y)$ is a $C^{2}$ solution of (4.9) due to $\nabla_{y}^{\alpha} \tilde{\phi}(0$, $\left.y_{2}\right)=\nabla_{y}^{\alpha} \phi\left(0, y_{2}\right)$ with $|\alpha|=2$.

Completely analogous to the first case, we can conclude that $\hat{\phi}(y)$ is analytic in $B\left(M_{0}, \delta\right)$. This also means that $\phi(y)$ is analytic in $B\left(M_{0}, \delta\right) \cap \tilde{\Omega}_{1}$. Thus, it is derived that $\phi\left(x_{3}, r\right)$ is analytic in $B\left(M_{0}, \delta\right) \cap \bar{\Omega}_{1}$.

Consequently, we complete the proof on Lemma 4.1 .

It is noted that corresponding to the transonic shock wave $r=\tilde{s}_{0} x_{3}$, it follows from the proof procedure of Lemma 2.1 that there exists a cone $r=\tilde{b}_{0} x_{3}$ such that the problem $(2.1)-(2.5)$ has a unique transonic solution $\left(\bar{\rho}(s), \bar{U}(s), \bar{u}_{3}(s), \bar{P}(s)\right)$ for $\tilde{b}_{0} \leq s \leq \tilde{s}_{0}$. We denote $\bar{\phi}\left(x_{3}, r\right)$ by the potential function of $\left(\bar{U}\left(r / x_{3}\right), \bar{u}_{3}\left(r / x_{3}\right)\right)$. Then $\bar{\phi}\left(x_{3}, r\right)$ satisfies

$$
\begin{cases}\left(c^{2}(\nabla \bar{\phi})-\left(\partial_{x_{3}} \bar{\phi}\right)^{2}\right) \partial_{x_{3}}^{2} \bar{\phi}-2 \partial_{x_{3}} \bar{\phi} \partial_{r} \bar{\phi} \partial_{r x_{3}}^{2} \bar{\phi} & \\ \quad+\left(c^{2}(\nabla \bar{\phi})-\left(\partial_{r} \bar{\phi}\right)^{2}\right) \partial_{r}^{2} \bar{\phi}+\frac{c^{2}(\nabla \bar{\phi})}{r} \partial_{r} \bar{\phi}=0 & \text { in } \Omega_{2}, \\ \bar{\phi}\left(x_{3}, r\right)=\left(u_{3}\left(\tilde{s}_{0}\right)+U\left(\tilde{s}_{0}\right) \tilde{s}_{0}\right) x_{3} & \text { on } r=\tilde{s}_{0} x_{3}, \\ \partial_{\vec{n}} \bar{\phi}\left(x_{3}, r\right)=\tilde{s}_{0} u_{3}\left(\tilde{s}_{0}\right)-U\left(\tilde{s}_{0}\right) & \text { on } r=\tilde{s}_{0} x_{3}, \\ \partial_{r} \bar{\phi}\left(x_{3}, r\right)=\tilde{b}_{0} \partial_{x_{3}} \bar{\phi}\left(x_{3}, r\right) & \text { on } r=\tilde{b}_{0} x_{3},\end{cases}
$$

where $\Omega_{2}=\left\{\left(x_{3}, r\right): \tilde{b}_{0} x_{3}<r<\tilde{s}_{0} x_{3}, x_{3}>0\right\}$.

Similar to the proof of Lemma 4.1, we have the following.

LEMMA 4.2. $\bar{\phi}\left(x_{3}, r\right)$ is analytic in $\bar{\Omega}_{2} \backslash\left\{\left(x_{3}, r\right): r=\tilde{b}_{0} x_{3}, x_{3} \geq 0\right\}$.

Next, we study the precise asymptotic state of the transonic shock solution at infinity, which will be used in the proof of Theorem 1.1. 
LEMMA 4.3. If the shock curve is straight and if the solution of (1.5)-(1.9) is globally stable, then $\left(\rho^{+}\left(x_{3}, r\right), U^{+}\left(x_{3}, r\right), u_{3}^{+}\left(x_{3}, r\right), P^{+}\left(x_{3}, r\right)\right)$ tends to a constant vector $\left(\rho_{\infty}, U_{\infty}, u_{3 \infty}, P_{\infty}\right)$ as $x_{3} \rightarrow \infty$ along each stream line.

Proof. It follows from condition (1.9) that the solution tends to a constant vector along each stream line when $x_{3} \rightarrow \infty$. However, in the general case, the related constant vectors are different along different stream lines. Fortunately, for the case of the straight shock, we can show that these constant vectors are the same. To see this, it is convenient to use the Lagrange transformation (3.3) to analyze our problem.

Denote $\lim _{\xi \rightarrow \infty}\left(\rho^{+}(\xi, \eta), U^{+}(\xi, \eta), u_{3}^{+}(\xi, \eta), P^{+}(\xi, \eta)\right)=\left(\rho_{\infty}(\eta), U_{\infty}(\eta)\right.$, $\left.u_{3 \infty}(\eta), P_{\infty}(\eta)\right)$. When $\xi \rightarrow \infty$, it follows from the second equation of $(3.5)$ and assumption Theorem 1.1(iii) that $\partial_{\eta} P_{\infty}(\eta)=0$ holds true, which implies that

$$
P_{\infty}(\eta)=\text { constant } \equiv P_{\infty}
$$

By (4.1), we arrive at

$$
\rho_{\infty}(\eta)=\text { constant } \equiv \rho_{\infty}
$$

Rewrite the first equation of (3.5) as follows:

$$
-\frac{1}{r^{2} \rho^{+} u_{3}^{+}} \partial_{\xi} r+\frac{1}{r} \partial_{\xi}\left(\frac{1}{\rho^{+} u_{3}^{+}}\right)-\partial_{\eta}\left(\frac{U^{+}}{u_{3}^{+}}\right)=0 .
$$

It is noted that $\lim _{\xi \rightarrow \infty} r(\xi, \eta)=\infty$ and $\partial_{\xi} r=U / u_{3}$; we can then derive from $(4.14)$ that $\partial_{\eta}\left(\left(U_{\infty}(\eta)\right) /\left(u_{3 \infty}(\eta)\right)\right)=0$ holds true as $\xi \rightarrow \infty$. This means that

$$
\frac{U_{\infty}(\eta)}{u_{3 \infty}(\eta)}=\text { constant }
$$

In addition, by use of the fourth equation in (3.5) and of (4.12) and (4.13), one has

$$
U_{\infty}^{2}(\eta)+u_{3 \infty}^{2}(\eta)=\text { constant }
$$

Combining (4.15) with (4.16) yields

$$
u_{3 \infty}(\eta)=\text { constant } \equiv u_{3 \infty}, \quad U_{\infty}(\eta)=\text { constant } \equiv U_{\infty} .
$$

Thus, we complete the proof of Lemma 4.3. 
We now show the following conclusion. From this, Theorem 1.1 will be easily derived.

THEOREM 4.1. Under the assumptions of Theorem 1.1, we can derive that $\Omega_{1}=\Omega_{2}=\Omega$ holds true, where $\Omega_{1}=\left\{\left(x_{3}, r\right): b\left(x_{3}\right)<r<\tilde{s}_{0} x_{3}, x_{3}>\right.$ $0\}, \Omega_{2}=\left\{\left(x_{3}, r\right): \tilde{b}_{0} x_{3}<r<\tilde{s}_{0} x_{3}, x_{3}>0\right\}$, and $\Omega=\left\{\left(x_{3}, r\right): b_{0} x_{3}<r<\right.$ $\left.s_{0} x_{3}, x_{3}>0\right\}$. Moreover, $\phi\left(x_{3}, r\right) \equiv \bar{\phi}\left(x_{3}, r\right)$, where $\bar{\phi}\left(x_{3}, r\right)$ is given in (4.11).

Proof. We divide the proof procedure into two steps.

Step 1. $\phi\left(x_{3}, r\right)=\bar{\phi}\left(x_{3}, r\right)$ holds in $\Omega_{1} \cap \Omega_{2}$. It follows from Lemmas 4.1 and 4.2 that $\phi\left(x_{3}, r\right)$ and $\bar{\phi}\left(x_{3}, r\right)$ both are analytic solutions of the Cauchy problem (4.4)-(4.6) in $\left(\Omega_{1} \cap \Omega_{2}\right) \cup \tilde{\Sigma}$, where $\tilde{\Sigma} \equiv\left\{\left(x_{3}, r\right): r=\tilde{s}_{0} x_{3}, x_{3}>0\right\}$. By the uniqueness of analytic solution in the Cauchy-Kowalewsky theorem, one knows that $\phi\left(x_{3}, r\right) \equiv \bar{\phi}\left(x_{3}, r\right)$ in $\Omega_{1} \cap \Omega_{2}$.

Step 2. $\Omega_{1} \equiv \Omega_{2}$. We will use the contradiction method to show this. Otherwise, if $\Omega_{1} \not \equiv \Omega_{2}$, then there will exist three possible cases:

(i) $\Omega_{2} \subset \Omega_{1}, \Gamma_{1} \cap \Gamma_{2}=\phi$;

(ii) $\Omega_{1} \subset \Omega_{2}, \Gamma_{1} \cap \Gamma_{2}=\phi$; and

(iii) the left case, $\Gamma_{1} \cap \Gamma_{2} \neq \phi$, where $\Gamma_{1}=\left\{\left(x_{3}, r\right): r=b\left(x_{3}\right), x_{3}>0\right\}$ and $\Gamma_{2}=\left\{\left(x_{3}, r\right): r=\tilde{b}_{0} x_{3}, x_{3}>0\right\}$.

In case $(\mathrm{i})$, one has $\tilde{b}_{0} x_{3}>b\left(x_{3}\right)$ (see Figure 5$)$.

It follows from Step 1 that

$$
\phi\left(x_{3}, r\right)=\bar{\phi}\left(x_{3}, r\right) \quad \text { in } \Omega_{2} .
$$

Along the stream line $r=\tilde{b}_{0} x_{3}$, we have

$$
\partial_{r} \phi\left(x_{3}, \tilde{b}_{0} x_{3}\right)=\partial_{r} \bar{\phi}\left(x_{3}, \tilde{b}_{0} x_{3}\right)=\tilde{b}_{0} \partial_{x_{3}} \bar{\phi}\left(x_{3}, \tilde{b}_{0} x_{3}\right)=\tilde{b}_{0} \partial_{x_{3}} \phi\left(x_{3}, \tilde{b}_{0} x_{3}\right) .
$$

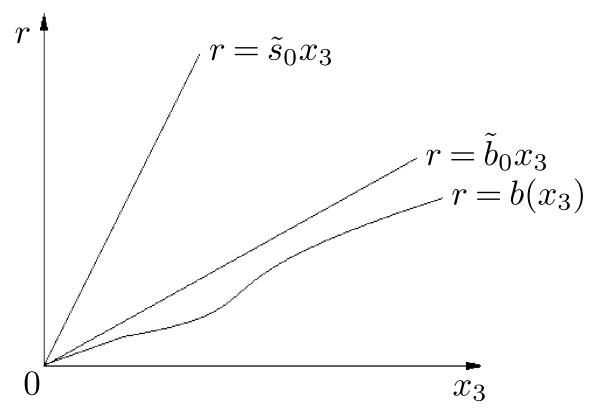

Figure 5 


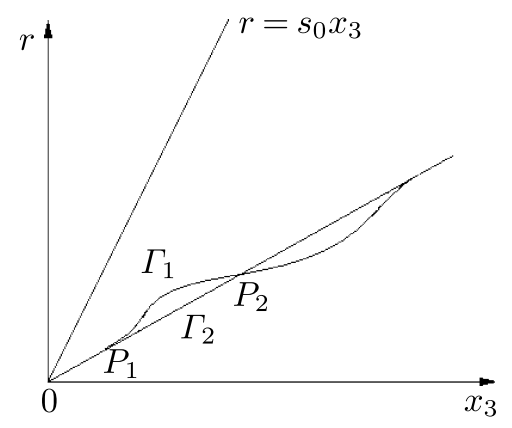

Figure 6

This implies that

$$
U^{+}\left(x_{3}, \tilde{b}_{0} x_{3}\right)=\tilde{b}_{0} u_{3}^{+}\left(x_{3}, \tilde{b}_{0} x_{3}\right) .
$$

By Lemma 4.3, we arrive at

$$
U_{\infty}=\tilde{b}_{0} u_{3 \infty}
$$

On the other hand, $r=b\left(x_{3}\right)$ is also a stream line; that is, $\partial_{r} \phi\left(x_{3}, b\left(x_{3}\right)\right)=$ $b^{\prime}\left(x_{3}\right) \partial_{x_{3}} \phi\left(x_{3}, b\left(x_{3}\right)\right)$. From this, Lemma 4.3 , and the fact $b^{\prime}\left(x_{3}\right)=b_{0}$ for large $x_{3}$, we have

$$
U_{\infty}=b_{0} u_{3 \infty}
$$

Combining (4.17) with (4.18) yields $b_{0}=\tilde{b}_{0}$. However, this is a contradiction with $\Gamma_{1} \cap \Gamma_{2}=\phi$. Thus, case (i) does not happen. Analogously, case (ii) does not happen.

Next, we consider case (iii) (see Figure 6).

Since $\tilde{b}_{0}=b_{0}$ holds true, then the boundary $\Gamma_{2}=\left\{\left(x_{3}, r\right): r=b_{0} x_{3}, x_{3}>\right.$ $0\}$ must intersect with $\Gamma_{1}$. Because of $b\left(x_{3}\right)=b_{0} x_{3}+\varepsilon \varphi\left(x_{3}\right)$ with $\varphi\left(x_{3}\right) \in$ $C_{0}^{\infty}(0, l)$, we then can conclude that there exist at least two intersection points $P_{1}$ and $P_{2}$ in $\Gamma_{1} \cap \Gamma_{2}$ such that the arc $P_{1} P_{2}$ lies above $\Gamma_{2}$ or lies below $\Gamma_{2}$. Without loss of generality, we assume that the arc $P_{1} P_{2}$ lies above $\Gamma_{2}$. In addition, for convenience, the coordinate of $P_{1}$ is denoted by $\left(x_{3}^{P_{1}}, r^{P_{1}}\right)$.

Consider the following stream line starting from $P_{1}$ :

$$
\left\{\begin{array}{l}
\frac{d r}{d x_{3}}=\frac{\partial_{r} \bar{\phi}\left(x_{3}, r\right)}{\partial_{x_{3}} \bar{\phi}\left(x_{3}, r\right)} \\
r\left(x_{3}^{P_{1}}\right)=r^{P_{1}}
\end{array}\right.
$$


Because of Step 1, we know that there exist two different stream lines $r=b_{0} x_{3}$ and $r=b\left(x_{3}\right)$ between $P_{1}$ and $P_{2}$ for the system (1.5). However, this is contradictory with the uniqueness of solution to the ordinary differential equation (4.19). Thus, we must have $\Omega_{1}=\Omega_{2}$ in order to guarantee the global stability of the transonic shock solution, and the proof of Theorem 4.1 is completed.

Based on Theorem 4.1, we now start to prove Theorem 1.1.

Proof of Theorem 1.1. By use of Theorem 4.1, $\Omega_{1} \equiv \Omega_{2} \equiv \Omega$ holds true. However, the boundary $r=b\left(x_{3}\right)$ is obviously different from the boundary $r=b_{0} x_{3}$. Therefore, under the assumptions of Theorem 1.1, a globally stable transonic shock solution does not exist.

\section{Appendix}

In this appendix, we show that the self-similar downstream subsonic state $\left(\tilde{\rho}\left(r / x_{3}\right), \tilde{U}\left(r / x_{3}\right), \tilde{u}_{3}\left(r / x_{3}\right), \tilde{P}\left(r / x_{3}\right)\right)$ behind the transonic shock $r=s_{0} x_{3}$ satisfies all assumptions in Theorem 1.1. Since assumptions (i) and (ii) hold true obviously, it suffices to prove assumption (iii).

Let us define the stream line $r=r\left(x_{3}, x_{3}^{0}\right)$ starting from the point $\left(x_{3}^{0}\right.$, $\left.s_{0} x_{3}^{0}\right)$ at the shock $r=s_{0} x_{3}$ as follows:

$$
\left\{\begin{array}{l}
\frac{d r\left(x_{3}, x_{3}^{0}\right)}{d x_{3}}=\left(\frac{\tilde{U}}{\tilde{u}_{3}}\right)\left(\frac{r\left(x_{3}, x_{3}^{0}\right)}{x_{3}}\right), \\
r\left(x_{3}^{0}, x_{3}^{0}\right)=s_{0} x_{3}^{0}
\end{array}\right.
$$

where $x_{3}^{0}>0$.

For notational convenience, we set $s=\left(r\left(x_{3}, x_{3}^{0}\right)\right) / x_{3}$. Then it follows from a direct computation that

$$
\frac{d}{d x_{3}}\left(s-\frac{\tilde{U}(s)}{\tilde{u}_{3}(s)}\right)=\frac{1}{x_{3}}\left(\frac{\tilde{U}}{\tilde{u}_{3}}-s\right)^{\prime}(s)\left(s-\frac{\tilde{U}}{\tilde{u}_{3}}\right) .
$$

By use of (2.1), we have

$$
\left(\frac{\tilde{U}}{\tilde{u}_{3}}-s\right)^{\prime}(s)=-1-\frac{c^{2}(s) \tilde{U}\left(s \tilde{U}+\tilde{u}_{3}\right)}{s \tilde{u}_{3}^{2}\left(\left(1+s^{2}\right) c^{2}(s)-\left(s \tilde{u}_{3}-\tilde{U}\right)^{2}\right)} .
$$

It follows from Lemma 2.1 and (A.3) that there exists a uniform positive constant $\delta$ such that

$$
\left(\frac{\tilde{U}}{\tilde{u}_{3}}-s\right)^{\prime}(s) \leq-1-\delta
$$


Because $s-(\tilde{U}(s)) /\left(\tilde{u}_{3}(s)\right) \geq 0$ for $b_{0} \leq s \leq s_{0}$ (see $\left.(2.21)\right)$, we then arrive at

$$
\frac{d}{d x_{3}}\left(s-\frac{\tilde{U}(s)}{\tilde{u}_{3}(s)}\right) \leq-\frac{1+\delta}{x_{3}}\left(s-\frac{\tilde{U}(s)}{\tilde{u}_{3}(s)}\right) .
$$

This implies that

$$
\frac{d}{d x_{3}}\left(x_{3}^{1+\delta}\left(s-\frac{\tilde{U}(s)}{\tilde{u}_{3}(s)}\right)\right) \leq 0 .
$$

Thus, one has

$$
s-\frac{\tilde{U}(s)}{\tilde{u}_{3}(s)} \leq \frac{C\left(x_{3}^{0}\right)}{x_{3}^{1+\delta}},
$$

where $C\left(x_{3}^{0}\right)$ is a positive constant depending on $x_{3}^{0}$.

Since for $\tilde{x}_{3}>X_{3}>x_{3}^{0}$ we have

$$
\begin{aligned}
\left|\frac{r\left(\tilde{x}_{3}, x_{3}^{0}\right)}{\tilde{x}_{3}}-\frac{r\left(X_{3}, x_{3}^{0}\right)}{X_{3}}\right| & =\left|\int_{X_{3}}^{\tilde{x}_{3}} \frac{d}{d x_{3}}\left(\frac{r\left(x_{3}, x_{3}^{0}\right)}{x_{3}}\right) d x_{3}\right|=\int_{X_{3}}^{\tilde{x}_{3}} \frac{1}{x_{3}}\left(s-\frac{\tilde{U}}{\tilde{u}_{3}}\right) d x_{3} \\
& \leq C\left(x_{3}^{0}\right) \int_{X_{3}}^{\tilde{x}_{3}} \frac{1}{x_{3}^{2+\delta}} d x_{3}=\frac{C\left(x_{3}^{0}\right)}{1+\delta}\left(\frac{1}{X_{3}^{1+\delta}}-\frac{1}{\tilde{x}_{3}^{1+\delta}}\right),
\end{aligned}
$$

one can then derive that

$$
\lim _{x_{3} \rightarrow \infty} \frac{r\left(x_{3}, x_{3}^{0}\right)}{x_{3}}=\mu\left(x_{3}^{0}\right) .
$$

Next, we further prove $\mu\left(x_{3}^{0}\right) \equiv b_{0}$ for any $x_{3}^{0}>0$. In fact, it follows from (A.6) and Lemma 2.1 that

$$
\lim _{x_{3} \rightarrow \infty}\left(s \tilde{u}_{3}(s)-\tilde{U}(s)\right)=0 .
$$

This, together with (A.7), yields

$$
\mu\left(x_{3}^{0}\right) \tilde{u}_{3}\left(\mu\left(x_{3}^{0}\right)\right)-\tilde{U}\left(\mu\left(x_{3}^{0}\right)\right)=0 .
$$

By (2.20) and (2.21), we can easily obtain

$$
\mu\left(x_{3}^{0}\right) \equiv b_{0}
$$


Therefore, along the stream line $r=r\left(x_{3}, x_{3}^{0}\right)$,

$$
\lim _{x_{3} \rightarrow \infty}\left(\tilde{\rho}, \tilde{U}, \tilde{u}_{3}, \tilde{P}\right)\left(\frac{r\left(x_{3}, x_{3}^{0}\right)}{x_{3}}\right)=\left(\tilde{\rho}, \tilde{U}, \tilde{u}_{3}, \tilde{P}\right)\left(b_{0}\right) .
$$

Additionally, a direct computation yields

$$
\begin{gathered}
\lim _{x_{3} \rightarrow \infty} \partial_{x_{3}}\left(\left(\tilde{\rho}, \tilde{U}, \tilde{u}_{3}, \tilde{P}\right)\left(\frac{r\left(x_{3}, x_{3}^{0}\right)}{x_{3}}\right)-\left(\tilde{\rho}, \tilde{U}, \tilde{u}_{3}, \tilde{P}\right)\left(b_{0}\right)\right) \\
\quad=\lim _{x_{3} \rightarrow \infty} \frac{1}{x_{3}}\left(\tilde{\rho}^{\prime}, \tilde{U}^{\prime}, \tilde{u}_{3}^{\prime}, \tilde{P}^{\prime}\right)(s)\left(\frac{\tilde{U}(s)}{\tilde{u}_{3}(s)}-s\right)=0 .
\end{gathered}
$$

In order to calculate

$$
\lim _{x_{3} \rightarrow \infty} \partial_{x_{3}^{0}}\left(\left(\tilde{\rho}, \tilde{U}, \tilde{u}_{3}, \tilde{P}\right)\left(\frac{r\left(x_{3}, x_{3}^{0}\right)}{x_{3}}\right)-\left(\tilde{\rho}, \tilde{U}, \tilde{u}_{3}, \tilde{P}\right)\left(b_{0}\right)\right),
$$

it is noted that $\left(d r\left(x_{3}, x_{3}^{0}\right)\right) /\left(d x_{3}^{0}\right)$ satisfies the following equation from (A.1):

$$
\left\{\begin{array}{l}
\frac{d}{d x_{3}}\left(\frac{d r\left(x_{3}, x_{3}^{0}\right)}{d x_{3}^{0}}\right)=\left(\frac{\tilde{U}}{\tilde{u}_{3}}\right)^{\prime}(s) \frac{1}{x_{3}} \frac{d r\left(x_{3}, x_{3}^{0}\right)}{d x_{3}^{0}}, \\
\left.\left(\frac{d r\left(x_{3}, x_{3}^{0}\right)}{d x_{3}^{0}}\right)\right|_{x_{3}=x_{3}^{0}}=s_{0}-\left(\frac{\tilde{U}}{\tilde{u}_{3}}\right)\left(s_{0}\right) .
\end{array}\right.
$$

Because

$$
\left(\frac{\tilde{U}}{\tilde{u}_{3}}\right)^{\prime}(s)=-\frac{c^{2}(s) \tilde{U}\left(s \tilde{U}+\tilde{u}_{3}\right)}{s \tilde{u}_{3}^{2}\left(\left(1+s^{2}\right) c^{2}(s)-\left(s \tilde{u}_{3}-\tilde{U}\right)^{2}\right)}<0
$$

and $s_{0}-\left(\tilde{U} / \tilde{u}_{3}\right)\left(s_{0}\right)>0$, we then arrive at $0<\frac{d r\left(x_{3}, x_{3}^{0}\right)}{d x_{3}^{0}} \leq s_{0}-\left(\frac{\tilde{U}}{\tilde{u}_{3}}\right)\left(s_{0}\right)$.

Thus,

$$
\begin{aligned}
& \quad \lim _{x_{3} \rightarrow \infty} \partial_{x_{3}^{0}}\left(\left(\tilde{\rho}, \tilde{U}, \tilde{u}_{3}, \tilde{P}\right)\left(\frac{r\left(x_{3}, x_{3}^{0}\right)}{x_{3}}\right)-\left(\tilde{\rho}, \tilde{U}, \tilde{u}_{3}, \tilde{P}\right)\left(b_{0}\right)\right) \\
& \quad=\lim _{x_{3} \rightarrow \infty} \frac{1}{x_{3}}\left(\tilde{\rho}^{\prime}, \tilde{U}^{\prime}, \tilde{u}_{3}^{\prime}, \tilde{P}^{\prime}\right)(s) \frac{d r\left(x_{3}, x_{3}^{0}\right)}{d x_{3}^{0}} \\
& \quad=0 .
\end{aligned}
$$

Combining (A.11) with (A.12) and (A.13), we know that assumption Theorem 1.1(iii) holds true for the transonic background solution. Then, Remark 1.1 is proved. 


\section{REFERENCES}

[1] F. Asakura, Global solutions with a single transonic shock wave for quasilinear hyperbolic systems, Methods Appl. Anal. 4 (1997), 33-52.

[2] S. Bernstein, Demonstration du théorème de M. Hilbert sur la nature analytique des solutions des équations du type elliptique sans l'emploi des séries normales, Math. Z. 28 (1928), 330-348.

[3] S. Canic, B. L. Keyfitz, and G. M. Lieberman, A proof of existence of perturbed steady transonic shocks via a free boundary problem, Comm. Pure Appl. Math. 53 (2000), 484-511.

[4] S. Chen, A free boundary value problem of Euler system arising in supersonic flow past a curved cone, Tohoku Math. J. (2) 54 (2002), 105-120.

[5] S. Chen, Z. Xin, and H. Yin, Global shock wave for the supersonic flow past a perturbed cone, Comm. Math. Phys. 228 (2002), 47-84.

[6] R. Courant and K. O. Friedrichs, Supersonic Flow and Shock Waves, Interscience, New York, 1948.

[7] D. Cui and H. Yin, Global conic shock wave for the steady supersonic flow past a cone: Isothermal case, Pacific J. Math. 233 (2008), 101-133.

[8] D. Cui and H. Yin, Global conic shock wave for the steady supersonic flow past a cone: Polytropic gas, J. Differential Equations 246 (2009), 641-669.

[9] A. Friedman, On the regularity of the solutions of nonlinear elliptic and parabolic systems of partial differential equations, J. Math. Mech. 7 (1958), 43-59.

[10] D. Gilbarg and N. S. Tudinger, Elliptic Partial Differential Equations of Second Order, 2nd ed., Grundlehren Math. Wiss. 224, Springer, Berlin, 1998.

[11] A. Kuz'min, Solvability of a problem for transonic flow with a local supersonic region, Nonlinear Differential Equations Appl. 8 (2001), 299-321.

[12] A. Kuz'min, Boundary-Value Problems for Transonic Flow, Wiley, West Sussex, U.K., 2002.

[13] W. C. Lien and T. P. Liu, Nonlinear stability of a self-similar 3-dimensional gas flow, Comm. Math. Phys. 204 (1999), 525-549.

[14] C. S. Morawetz, On the nonexistence of continuous transonic flows past profiles, I, Comm. Pure Appl. Math. 9 (1956), 45-68; III, 11 (1958), 129-144; 17 (1964), 357-367; 10 (1975), 107-132.

[15] Z. Xin and H. Yin, Global multidimensional shock wave for the steady supersonic flow past a three-dimensional curved cone, Anal. Appl. 4 (2006), 101-132.

[16] G. Xu and H. Yin, Global transonic conic shock wave for the symmetrically perturbed supersonic flow past a cone, J. Differential Equations 245 (2008), 3389-3432.

[17] G. Xu and H. Yin, Global multidimensional transonic conic shock wave for the perturbed supersonic flow past a cone, SIAM J. Math. Anal. 41 (2009), 178-218.

[18] H. Yin and C. Zhou, On global transonic shocks for the steady supersonic Euler flows past sharp 2-D wedges, J. Differential Equations 246 (2009), 4466-4496.

[19] Y. Zheng, A global solution to a two-dimensional Riemann problem involving shocks as free boundaries, Acta Math. Appl. Sin. Engl. Ser. 19 (2003), 559-572. 


\section{Gang Xu}

Department of Mathematics and Institute of Mathematical Sciences Nanjing University

Nanjing 210093

China

gxu@ujs.edu.cn

Huicheng Yin

Department of Mathematics and Institute of Mathematical Sciences Nanjing University

Nanjing 210093

China

huicheng@nju.edu.cn 